UCSD/PTH 00-28

hep-ph/0011336

\title{
An effective field theory for collinear and soft gluons: heavy to light decays
}

\author{
Christian W. Bauer ${ }^{a}$, Sean Fleming ${ }^{b}$, Dan Pirjol ${ }^{a}$, and Iain W. Stewart ${ }^{a}$ \\ a Physics Department, University of California at San Diego, La Jolla, CA 92093 \\ ${ }^{b}$ Physics Department, Carnegie Mellon University, Pittsburgh, PA 15213
}

\begin{abstract}
We construct the Lagrangian for an effective theory of highly energetic quarks with energy $Q$, interacting with collinear and soft gluons. This theory has two low energy scales, the transverse momentum of the collinear particles, $p_{\perp}$, and the scale $p_{\perp}^{2} / Q$. The heavy to light currents are matched onto operators in the effective theory at one-loop and the renormalization group equations for the corresponding Wilson coefficients are solved. This running is used to sum Sudakov logarithms in inclusive $B \rightarrow X_{s} \gamma$ and $B \rightarrow X_{u} \ell \bar{\nu}$ decays. We also show that the interactions with collinear gluons preserve the relations for the soft part of the form factors for heavy to light decays found by Charles et al., establishing these relations in the large energy limit of QCD.
\end{abstract}




\section{INTRODUCTION}

The phenomenology of hadrons containing a single heavy quark is greatly simplified by the fact that nonperturbative hadronic physics can be parameterized by an expansion in $\Lambda_{\mathrm{QCD}} / m$, where $m$ is the mass of the heavy quark. At lowest order, interactions are insensitive to the heavy quark mass and spin, leading to new spin-flavor symmetries [1]. These symmetries relate form factors for decays of one heavy hadron to another heavy hadron. In Ref. [1,2] Heavy Quark Effective Theory (HQET) was constructed as a general framework in which to explore heavy quark physics. The effective theory allows a systematic treatment of $1 / m$ corrections and makes the symmetries manifest. Inclusive decays of heavy hadrons involving large momentum transfer to the decay products can also be treated in HQET with the help of the operator product expansion (OPE) [3]. At leading order the parton model results are recovered, and nonperturbative corrections are parameterized by matrix elements of higher dimensional operators, suppressed by powers of $1 / \mathrm{m}$.

Decays of heavy hadrons to light hadrons cannot be treated exclusively with HQET unless the four-momentum of the light degrees of freedom are small compared to $m$. However, in regions of phase space where the light hadronic decay products have large energy $E \sim m$, a different expansion in powers of $1 / E$ can be performed. In Ref. [4] Dugan and Grinstein used this approach to construct the large energy effective theory (LEET), which describes the interaction of very energetic quarks with soft gluons. However, LEET is missing an important degree of freedom, namely collinear gluons, and does not reproduce the IR physics of QCD [5]. In Ref. [6] it was shown that an effective theory including both collinear and soft gluons correctly reproduces the infrared physics of QCD at one loop. This collinear-soft theory is needed between the scale $E$ and an intermediate scale, below which collinear modes

can be integrated out. For inclusive decays it was shown that the collinear-soft theory can be matched at the intermediate scale onto a theory containing only soft degrees of freedom.

The power counting in the collinear-soft theory is complicated by the presence of two low energy scales, which must be properly accounted for. These scales can be clearly seen by considering the momentum of a collinear quark. If the quark moves along the light cone direction $n^{\mu}$ with momentum $Q \sim E \sim m$ then $p=\left(p^{+}, p^{-}, p_{\perp}\right) \sim Q\left(\lambda^{2}, 1, \lambda\right)$, where $\lambda$ is a small parameter. Thus $p_{\perp} \sim Q \lambda$ is the intermediate scale. With two low energy scales it is more appropriate to count powers of $\lambda$ rather than powers of $1 / Q$ [6]. This is analogous to Non-Relativistic QCD (NRQCD) for bound states of two heavy quarks, where one counts powers of the velocity rather than powers of $1 / m[7]$. Constructing such an effective field theory bears some similarity to isolating momentum regimes using the method of regions [8] on full theory Feynman diagrams. There are, however, advantages to using an effective field theory approach over the method of regions; namely it is straightforward to systematically 
include power corrections, and it is possible to properly account for operator running, which sums Sudakov logarithms. In order to consistently go beyond leading order it is important to give a detailed construction of the effective field theory. This was not done in Ref. [6], and it is one of the main points of this paper.

The collinear-soft effective theory can be used to describe both inclusive and exclusive heavy-to-light decays. For inclusive decays this theory is valid in the regime where the phase space of the decay is restricted such that the final hadronic state is forced to have low invariant mass and large energy. This is the case for large electron energy or small hadronic invariant mass in semileptonic $B \rightarrow X_{u} \ell \bar{\nu}$ decays, and for large photon energy in $B \rightarrow X_{s} \gamma$ decays. The Sudakov logarithms that appear in the endpoint regions of these decays can be summed into the coefficient function of operators by running in the collinear-soft theory between the scale $Q$ and $Q \lambda$, and then running a soft operator from $Q \lambda$ to $Q \lambda^{2}$. In Ref. [6] Sudakov logarithms at the endpoint of the photon energy spectrum in the decay $B \rightarrow X_{s} \gamma$ were summed in this manner. Here we sum Sudakov logarithms between $Q$ and $Q \lambda$ for both $B \rightarrow X_{s} \gamma$ and $B \rightarrow X_{u} \ell \bar{\nu}$. In the ratio of large moments of these decay rates effects of physics below the intermediate scale cancel, and we reproduce previous calculations carried out using the factorization formalism [9,10].

It is also possible to apply the collinear-soft effective theory to exclusive heavy-to-light decays. The form factors for such transitions have contributions from the exchange of soft gluons with spectators (soft contributions), as well as from the exchange of collinear gluons (so called hard contributions). In this paper we will only consider the soft form factors, even though the two types of contributions are believed to be the same order in $1 / m_{b}$ [11 113. In Ref. [12] relations among the soft form factors were derived, and it was shown that only three independent functions are needed to describe heavy-to-light decays. However, these relations were obtained within the framework of LEET, which does not include collinear gluons. In this paper we show that the inclusion of collinear modes does not alter the soft form factor relations to leading order in $\lambda$. Since the collinear-soft effective theory reproduces the infrared physics of QCD at large energies, this establishes these soft form factor relations in the large energy limit of QCD.

In this paper we give a detailed construction of the collinear-soft effective theory and apply it to general heavy to light decays. In section II the Lagrangian for collinear gluons and collinear quarks is constructed and the Feynman rules are given. The power counting for collinear gluons is formulated in a gauge invariant way. The collinear-soft effective theory does not have the same spin symmetry as LEET, but is still invariant under a helicity transformation. In section III we construct the heavy to light currents in the effective theory at lowest order in $\lambda$. At this order the effective theory current couples to an arbitrary 
number of collinear gluons with a universal Wilson coefficient. The one-loop matching for the Wilson coefficients are then derived. In section IV the renormalization group evolution of these coefficients are computed. Finally, in section $\mathrm{V}$ we present two applications of this effective theory. First we sum Sudakov logarithms in the ratio of large moments of $B \rightarrow X_{s} \gamma$ and $B \rightarrow X_{u} \ell \bar{\nu}$ decay rates. Next we show that in the collinear-soft theory only three independent soft form factors describe exclusive heavy to light decays, establishing these form factor relations in the large energy limit of QCD. The one loop matching onto currents in the effective theory allows us to calculate the perturbative corrections to these form factor relations in an infrared safe manner. For the ratio of full theory form factors these hard corrections agree with Ref. [13].

\section{THE EFFECTIVE THEORY}

Decays of heavy hadrons to highly energetic light hadrons are most conveniently studied in the rest frame of the heavy hadron. In this reference frame the light particles move close to the light cone direction $n^{\mu}$ and their dynamics is best described in terms of light cone coordinates $p=\left(p^{+}, p^{-}, p_{\perp}\right)$, where $p^{+}=n \cdot p, p^{-}=\bar{n} \cdot p$. For motion in the $z$ direction we take $n^{\mu}=(1,0,0,-1)$ and $\bar{n}^{\mu}=(1,0,0,1)$, so $\bar{n} \cdot n=2$. For large energies the different light cone components are widely separated, with $p^{-} \sim Q$ being large, while $p_{\perp}$ and $p^{+}$are small. Taking the small parameter to be $\lambda \sim p_{\perp} / p^{-}$we have

$$
p^{\mu}=\bar{n} \cdot p \frac{n^{\mu}}{2}+\left(p_{\perp}\right)^{\mu}+n \cdot p \frac{\bar{n}^{\mu}}{2}=\mathcal{O}\left(\lambda^{0}\right)+\mathcal{O}\left(\lambda^{1}\right)+\mathcal{O}\left(\lambda^{2}\right)
$$

where we have used $p^{+} p^{-} \sim p_{\perp}^{2} \sim \lambda^{2}$ for fluctuations near the mass shell. The collinear quark can emit either a soft gluon or a gluon collinear to the large momentum direction and still stay near its mass shell. Collinear and soft gluons have light cone momenta that scale like $k_{c}=Q\left(\lambda^{2}, 1, \lambda\right)$ and $k_{s}=Q\left(\lambda^{2}, \lambda^{2}, \lambda^{2}\right)$, respectively. For scales above the typical off-shellness of the collinear degrees of freedom, $k_{c}^{2} \sim(Q \lambda)^{2}$, both gluon modes are required to correctly reproduce all the infrared physics of the full theory. This was described in [6], where it was shown that at a scale $\mu \sim Q$ QCD can be matched onto an effective theory that contains heavy quarks and light collinear quarks, as well as soft and collinear gluons.

The Lagrangian describing the interaction of collinear quarks with soft and collinear gluons can be obtained at tree level by expanding the full theory Lagrangian in powers of $\lambda$. We start from the QCD Lagrangian for massless quarks and gluons

$$
\mathcal{L}_{\mathrm{QCD}}=\bar{\psi} i \not D \psi-\frac{1}{4} G_{\mu \nu} G^{\mu \nu}
$$

where the covariant derivative is $D_{\mu}=\partial_{\mu}-i g T^{a} A_{\mu}^{a}$, and $G_{\mu \nu}$ is the gluon field strength. We begin by removing the large momenta from the effective theory fields, similar to the 
construction of HQET [2]. In HQET there are two relevant momentum scales, the mass of the heavy quark $m$ and $\Lambda_{\mathrm{QCD}}$. The scale $m$ is separated from $\Lambda_{\mathrm{QCD}}$ by writing $p=m v+k$, where $v^{2}=1$ and the residual momentum $k \ll m$. The variable $v$ becomes a label on the effective theory fields. Our case is slightly more complicated because there are three scales to consider. We split the momenta $p$ by taking

$$
p=\tilde{p}+k, \quad \text { where } \quad \tilde{p} \equiv \frac{1}{2}(\bar{n} \cdot p) n+p_{\perp} .
$$

The "large" parts of the quark momentum $\bar{n} \cdot p \sim 1$ and $p_{\perp} \sim \lambda$, denoted by $\tilde{p}$, become a label on the effective theory field, while the residual momentum $k^{\mu} \sim \lambda^{2}$ is dynamical. This is analogous to NRQCD where there are also three relevant scales $m, m \beta$, and $m \beta^{2}$ (and $\beta \ll 1$ is the $q \bar{q}$ bound state velocity). In NRQCD the three scales can be separated [0] by writing $P=(m, \overrightarrow{0})+p+k$ where $p \sim m \beta$ and the residual momentum $k \sim m \beta^{2}$. In this case both the momenta of order $m$ (i.e. $(1, \overrightarrow{0})$ ), and the momentum of order $m \beta$ are labels on the effective theory fields.

The large momenta $\tilde{p}$ are removed by defining a new field $\psi_{n, p}$ by

$$
\psi(x)=\sum_{\tilde{p}} e^{-i \tilde{p} \cdot x} \psi_{n, p} .
$$

A label $p$ is given to the $\psi_{n, p}$ field, with the understanding that only the components $\bar{n} \cdot p$ and $p_{\perp}$ are true labels. Derivatives $\partial^{\mu}$ on the field $\psi_{n, p}(x)$ give order $\lambda^{2}$ contributions. For a particle moving along the $n^{\mu}$ direction, the four component field $\psi_{n, p}$ has two large components $\xi_{n, p}$ and two small components $\xi_{\bar{n}, p}$. These components can be obtained from the field $\psi_{n, p}$ using projection operators

$$
\xi_{n, p}=\frac{\not h \bar{n}}{4} \psi_{n, p}, \quad \xi_{\bar{n}, p}=\frac{\not h n}{4} \psi_{n, p},
$$

and satisfy the relations

$$
\begin{array}{ll}
\frac{\not h \not h}{4} \xi_{n, p}=\xi_{n, p}, & \not h \xi_{n, p}=0, \\
\frac{\not h n}{4} \xi_{\bar{n}, p}=\xi_{\bar{n}, p}, & \not h \xi_{\bar{n}, p}=0 .
\end{array}
$$

In terms of these fields the quark part of the Lagrangian in Eq. (2) becomes

$$
\begin{array}{r}
\mathcal{L}=\sum_{\tilde{p}, \tilde{p}^{\prime}}\left[\bar{\xi}_{n, p^{\prime}} \frac{\not h}{2}(i n \cdot D) \xi_{n, p}+\bar{\xi}_{\bar{n}, p^{\prime}} \frac{\not h}{2}(\bar{n} \cdot p+i \bar{n} \cdot D) \xi_{\bar{n}, p}\right. \\
\left.+\bar{\xi}_{n, p^{\prime}}\left(\not p_{\perp}+i \not D_{\perp}\right) \xi_{\bar{n}, p}+\bar{\xi}_{\bar{n}, p^{\prime}}\left(p_{\perp}+i \not D_{\perp}\right) \xi_{n, p}\right] .
\end{array}
$$

Since the derivatives on the fermionic fields yield momenta of order $k \sim \lambda^{2}$ they are suppressed relative to the labels $\bar{n} \cdot p$ and $p_{\perp}$. Without the $\bar{n} \cdot D$ and $D_{\perp}$ derivatives, $\xi_{\bar{n}, p}$ is not a dynamical field. Thus, we can eliminate $\xi_{\bar{n}, p}$ at tree level by using the equation of motion 


\begin{tabular}{l|c|c|c|ccc}
\hline \hline & heavy quark & collinear quark & soft gluon & \multicolumn{3}{|c}{ collinear gluons } \\
\hline Field & $h_{v}$ & $\xi_{n, p}$ & $A_{s}^{\mu}$ & $\bar{n} \cdot A_{n, q}$ & $n \cdot A_{n, q}$ & $A_{n, q}^{\perp}$ \\
Scaling & $\lambda^{3}$ & $\lambda$ & $\lambda^{2}$ & $\lambda^{0}$ & $\lambda^{2}$ & $\lambda$ \\
\hline \hline
\end{tabular}

TABLE I. Power counting for the effective theory fields.

$$
(\bar{n} \cdot p+\bar{n} \cdot i D) \xi_{\bar{n}, p}=\left(\not p_{\perp}+i \not D_{\perp}\right) \frac{\not h}{2} \xi_{n, p}
$$

This is similar to the approach taken in QCD quantized on the light cone 14 and in QCD in the infinite momentum frame [15], where two components of the fermion field are constrained auxiliary fields and are thus removed from the theory. Eqs. (7) and (8) result in a Lagrangian involving only the two components $\xi_{n, p}:$ 円

$$
\mathcal{L}=\sum_{\tilde{p}, \tilde{p}^{\prime}} e^{-i\left(\tilde{p}-\tilde{p}^{\prime}\right) \cdot x} \bar{\xi}_{n, p^{\prime}}\left[n \cdot i D+\left(\not p_{\perp}+i \not D_{\perp}\right) \frac{1}{\bar{n} \cdot p+\bar{n} \cdot i D}\left(\not p_{\perp}+i \not D_{\perp}\right)\right] \frac{\not h}{2} \xi_{n, p} .
$$

Here the summation extends over all distinct copies of the fields labelled by $\tilde{p}, \tilde{p}$. Note that the gluon field in $D^{\mu}$ includes collinear and soft parts, $A^{\mu} \rightarrow A_{c}^{\mu}+A_{s}^{\mu}$. The two types of gluons are distinguished by the length scales over which they fluctuate. Fluctuations of the collinear gluon fields $A_{c}^{\mu}$ are characterized by the scale $q^{2} \sim \lambda^{2}$, while fluctuations of the soft gluon field $A_{s}^{\mu}$ are characterized by $k^{2} \sim \lambda^{4}$. Since the collinear gluon field has large momentum components $\tilde{q} \equiv\left(\bar{n} \cdot q, q_{\perp}\right)$, derivatives acting on these fields can still give order $\lambda^{0,1}$ contributions. To make this explicit we label the collinear gluon field by its large momentum components $\tilde{q}$, and extract the phase factor containing $\tilde{q}$ by redefining the field: $A_{c}(x) \rightarrow e^{-i \tilde{q} \cdot x} A_{n, q}(x)$. Inserting this into Eq. (9) one finds

$$
\begin{array}{r}
\mathcal{L}=\sum_{\tilde{p}, \tilde{p}^{\prime}, \tilde{q}} e^{-i\left(\tilde{p}-\tilde{p}^{\prime}\right) \cdot x} \bar{\xi}_{n, p^{\prime}}\left[n \cdot i D+g e^{-i \tilde{q} \cdot x} n \cdot A_{n, q}+\left(\not p_{\perp}+i \not D_{\perp}+g e^{-i \tilde{q} \cdot x} A_{n, q}^{\perp}\right)\right. \\
\left.\quad \times \frac{1}{\bar{n} \cdot p+\bar{n} \cdot i D+g e^{-i \tilde{q} \cdot x} \bar{n} \cdot A_{n, q}}\left(\not p_{\perp}+i \not D_{\perp}+g e^{-i \tilde{q} \cdot x} A_{n, q}^{\perp}\right)\right] \frac{\not h}{2} \xi_{n, p} .
\end{array}
$$

Here the covariant derivative is defined to only involve soft gluons.

\footnotetext{
${ }^{1}$ Note that Eq. (9) still includes particle/antiparticle and the two spin degrees of freedom. However, on the light cone the spinor for a spin-up (down) particle is identical to that of the spin-up (down) anti-particle. See for example, Ref. [15].
} 
Finally, we expand Eq. (10) in powers of $\lambda$. To simplify the power counting we follow the procedure [16] of moving all the dependence on $\lambda$ into the interaction terms of the action to make the kinetic terms of order $\lambda^{0}$. This is done by assigning a $\lambda$ scaling to the effective theory fields as given in Table [. The power counting in Table 《 gives an order one kinetic term for collinear gluons in an arbitrary gauge. In generalized covariant gauge

$$
\int d^{4} x e^{i k \cdot x}\left\langle 0\left|T A_{c}^{\mu}(x) A_{c}^{\nu}(0)\right| 0\right\rangle=\frac{-i}{k^{2}}\left(g^{\mu \nu}-\alpha \frac{k^{\mu} k^{\nu}}{k^{2}}\right)
$$

and the scaling of the components on the right and left hand side of this equation agree.[ With this power counting all interactions scale as $\lambda^{n}$ with $n \geq 0$. Expanding Eq. (10) to order $\lambda^{0}$ gives

$$
\begin{aligned}
\mathcal{L}_{c s}= & \bar{\xi}_{n, p}\left(n \cdot i D+\frac{p_{\perp}^{2}}{\bar{n} \cdot p}\right) \frac{\not h}{2} \xi_{n, p} \\
& +\bar{\xi}_{n, p+q}\left[g n \cdot A_{n, q}+g A_{n, q}^{\perp} \frac{p_{\perp}}{\bar{n} \cdot p}+\frac{\not p_{\perp}+\not q_{\perp}}{\bar{n} \cdot(p+q)} g A_{n, q}^{\perp}-\frac{\not p_{\perp}+\not q_{\perp}}{\bar{n} \cdot(p+q)} g \bar{n} \cdot A_{n, q} \frac{\not p_{\perp}}{\bar{n} \cdot p}\right] \frac{\not \hbar}{2} \xi_{n, p} \\
& +\ldots+\mathcal{O}(\lambda) .
\end{aligned}
$$

Summation over the labels $\tilde{p}, \tilde{q}$ is understood implicitly. The ellipsis denote terms of the same order in the power counting with two or more collinear gluon fields, and arise because we expanded Eq. (10) in powers of $g A_{c}$ to obtain the above expression. This expansion was necessary to move the collinear gluon phase factor appearing in the denominator of Eq. (10) into the numerator. This allowed us to remove the large momentum $\tilde{q}$ from the Lagrangian so that all covariant derivatives were truly of $\mathcal{O}\left(\lambda^{2}\right)$. The method for including terms of higher order in $\lambda$ should be obvious from our derivation. The first few Feynman rules which follow from the $\lambda^{0}$ terms in $\mathcal{L}_{c s}$ are shown in Fig. 1.

The first term in Eq. (12) gives the propagator for the collinear quarks, which does not change depending on whether it interacts with soft or collinear gluons. This is distinct from the situation in the method of regions [8], where one must determine the propagator on a case by case basis. The interaction with a soft gluon is obtained from the covariant derivative term in Eq. (12). Also shown in Fig. 1 are the interactions with one and two collinear gluons. The collinear gluon interactions are label changing unlike the interaction involving soft gluons. Since $\bar{n} \cdot A_{n, q} \sim \bar{n} \cdot p$, Eq. (12) includes interactions of a collinear quark with an arbitrary number of collinear gluons. In Fig. 1 only interactions through $\mathcal{O}\left(g^{2}\right)$ are

\footnotetext{
${ }^{2}$ We have chosen a different counting for the collinear gluon fields than Ref. [6] (where $\left.A_{c}^{\mu} \sim \lambda\right)$. In Feynman gauge there is the freedom to choose any scaling with $A_{c}^{+} A_{c}^{-} \sim \lambda^{2}$ (including the choice as in Ref. [6]). The choice in Table $\mathbb{1}$ is preferred since $A_{c}^{\mu}$ scales the same way as a collinear momentum and there are no interactions that scale as $1 / \lambda$.
} 


$$
\begin{aligned}
& \underset{(\tilde{p}, k)}{(}=i \frac{\not h}{2} \frac{\bar{n} \cdot p}{n \cdot k \bar{n} \cdot p+p_{\perp}^{2}+i \epsilon} \\
& \begin{array}{l}
\mu, \mathrm{A} \\
\text { فे }
\end{array}
\end{aligned}
$$

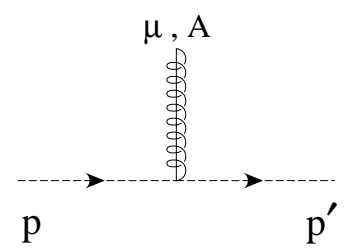

$$
\begin{aligned}
& =i g T^{A}\left[n_{\mu}+\frac{\gamma_{\mu}^{\perp} p_{\perp}}{\bar{n} \cdot p}+\frac{\not p_{\perp}^{\prime} \gamma_{\mu}^{\perp}}{\bar{n} \cdot p^{\prime}}-\frac{\not{ }_{\perp}^{\prime} p_{\perp}}{\bar{n} \cdot p \bar{n} \cdot p^{\prime}} \bar{n}_{\mu}\right] \frac{\not h}{2}
\end{aligned}
$$

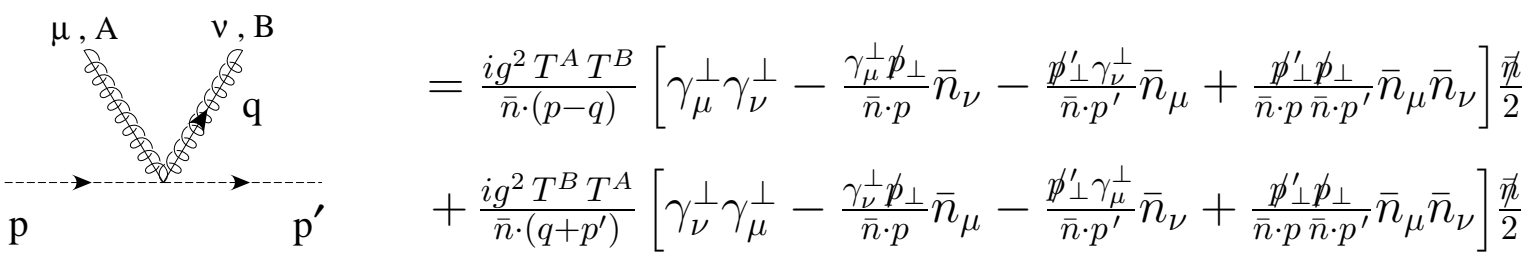

FIG. 1. Order $\lambda^{0}$ Feynman rules: collinear quark propagator with label $\tilde{p}$ and residual momentum $k$, and collinear quark interactions with one soft gluon, one collinear gluon, and two collinear gluons respectively.

shown. Note that in the light-cone gauge $\bar{n} \cdot A_{n, q}=0$ these Feynman rules are the complete set, since interactions of a collinear quark with three or more collinear gluons vanish. In this gauge similar Feynman rules for collinear gluons have been obtained in the framework of light cone QCD [17]. However, the Feynman rules in Fig. 11 can be used in any gauge.

The LEET Lagrangian corresponds to the $\bar{\xi}_{n, p} \frac{\vec{n}}{2} n \cdot i D \xi_{n, p}$ term in Eq. (12) and is invariant under a $\mathrm{SU}(2)$ symmetry 4,12 with generators $S^{1}=\left(\gamma^{0} \Sigma^{1}\right) / 2, S^{2}=\left(\gamma^{0} \Sigma^{2}\right) / 2$, and $S^{3}=$ $\Sigma^{3} / 2$ where $\Sigma^{i}$ are the standard rotation generators. The collinear soft Lagrangian $\mathcal{L}_{c s}$ has less symmetry than LEET because terms with $\gamma_{\perp}^{1} \gamma_{\perp}^{2}$ violate the transformations generated by $S^{1}$ and $S^{2}$. However, $\mathcal{L}_{c s}$ is still invariant under a $U(1)$, namely the helicity transformations generated by $S^{3}$. Since $S^{3}=\gamma^{5}(1 / 2-\not h \pitchfork / 4)$ and $\not h \xi_{n, p}=0$ the helicity transformation also corresponds to the chiral transformation generated by $\gamma^{5} / 2$.

To complete the construction of the effective theory we have to include heavy quarks. This can be done by adding the usual HQET Lagrangian for the heavy quark field $h_{v}$,

$$
\mathcal{L}_{\mathrm{HQET}}=\bar{h}_{v} i v \cdot D h_{v}
$$

The covariant derivative in Eq. (13) contains only the soft gluon field because the heavy 

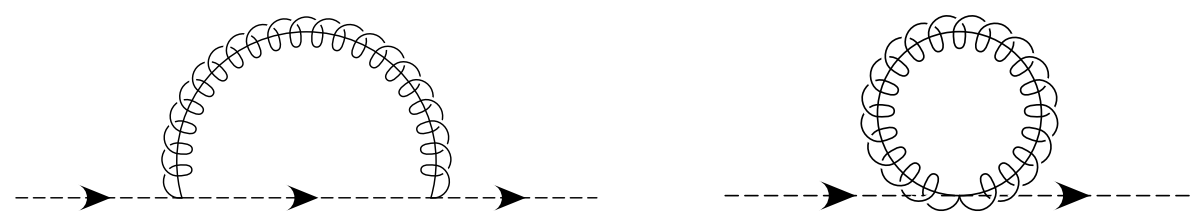

FIG. 2. Order $\alpha_{s} \lambda^{0}$ self energy diagrams for a collinear quark.

quark field does not couple to collinear gluons [6]. This is discussed in more detail in the next section.

As a simple application of the Feynman rules consider the order $\lambda^{0}$ diagrams for the self energy of a collinear quark shown in Fig. 2. The tadpole diagram vanishes in dimensional regularization. In Feynman gauge the remaining diagram gives

$$
\begin{aligned}
i \Sigma_{c}(p) & =g^{2} C_{F} \frac{\not h}{2} \int \frac{d^{d} l}{(2 \pi)^{d}}\left\{(n \cdot \bar{n}) \frac{p_{\perp}^{2}+\not p_{\perp} l_{\perp}}{\bar{n} \cdot p(p+l)^{2} l^{2}}+(n \cdot \bar{n}) \frac{p_{\perp}^{2}+l_{\perp} p_{\perp}}{\bar{n} \cdot p(p+l)^{2} l^{2}}\right. \\
& \left.+2(d-4) \frac{p_{\perp}^{2}+l_{\perp} \cdot p_{\perp}}{\bar{n} \cdot p(p+l)^{2} l^{2}}-(d-2)\left(\frac{\left(p_{\perp}+l_{\perp}\right)^{2}}{[\bar{n} \cdot(p+l)]^{2}}+\frac{p_{\perp}^{2}}{[\bar{n} \cdot p]^{2}}\right) \frac{\bar{n} \cdot(p+l)}{(p+l)^{2} l^{2}}\right\} .
\end{aligned}
$$

Here sums over the labels $\bar{n} \cdot l$ and $l_{\perp}$ were combined with the integrals over residual momenta to give the full $d^{d} l$ measure (c.f., Ref. [7]). The first two terms in Eq. (14) correspond to the $(\mu, \nu)=(+,-)$ and $(-,+)$ polarizations of the exchanged gluon, and the last line to the $(\perp, \perp)$ contribution, respectively. Computing the loop integrals one finds

$$
\begin{aligned}
& i \Sigma_{+-}(p)+i \Sigma_{-+}(p)=\frac{i \alpha_{s} C_{F}}{4 \pi} \frac{\not h}{2} \Gamma(\epsilon) \frac{\Gamma^{2}(1-\epsilon)}{\Gamma(2-2 \epsilon)} \frac{2 p_{\perp}^{2}}{\bar{n} \cdot p}\left(\frac{-p^{2}}{e^{\gamma_{E}} \mu^{2}}\right)^{-\epsilon} \\
& i \Sigma_{\perp \perp}(p)=-\frac{i \alpha_{s} C_{F}}{4 \pi} \frac{\not h}{2} \Gamma(\epsilon) \frac{\Gamma^{2}(1-\epsilon)}{\Gamma(2-2 \epsilon)}\left\{(1+\epsilon) \frac{p_{\perp}^{2}}{\bar{n} \cdot p}-(1-\epsilon) n \cdot p\right\}\left(\frac{-p^{2}}{e^{\gamma_{E}} \mu^{2}}\right)^{-\epsilon} .
\end{aligned}
$$

Here (and in the rest of the paper) we use $\overline{\mathrm{MS}}$ and therefore redefined $\mu^{2} \rightarrow \mu^{2} e^{\gamma_{E}} /(4 \pi)$. The sum has precisely the form of the inverse collinear quark propagator in Fig. 1:

$$
\Sigma_{c}(p)=\frac{\alpha_{s} C_{F}}{4 \pi} \frac{\not h}{2}(1-\epsilon) \Gamma(\epsilon) \frac{\Gamma^{2}(1-\epsilon)}{\Gamma(2-2 \epsilon)} \frac{p^{2}}{\bar{n} \cdot p}\left(\frac{-p^{2}}{e^{\gamma_{E}} \mu^{2}}\right)^{-\epsilon} .
$$

The ultraviolet divergence in this expression is removed by onshell wavefunction renormalization of the effective theory field $\xi_{n, p}$,

$$
Z_{\xi}=1-\frac{\alpha_{s} C_{F}}{4 \pi}\left[\frac{1}{\epsilon}-\log \left(\frac{-p^{2}}{\mu^{2}}\right)+1\right] .
$$

$Z_{\xi}$ coincides with the renormalization of the quark field in QCD. This is expected [6] since without currents or soft effects the collinear quark Lagrangian simply describes QCD in a particular frame. The utility of the two component formalism in Eq. (12) will become evident in the next section where heavy to light currents are discussed. 


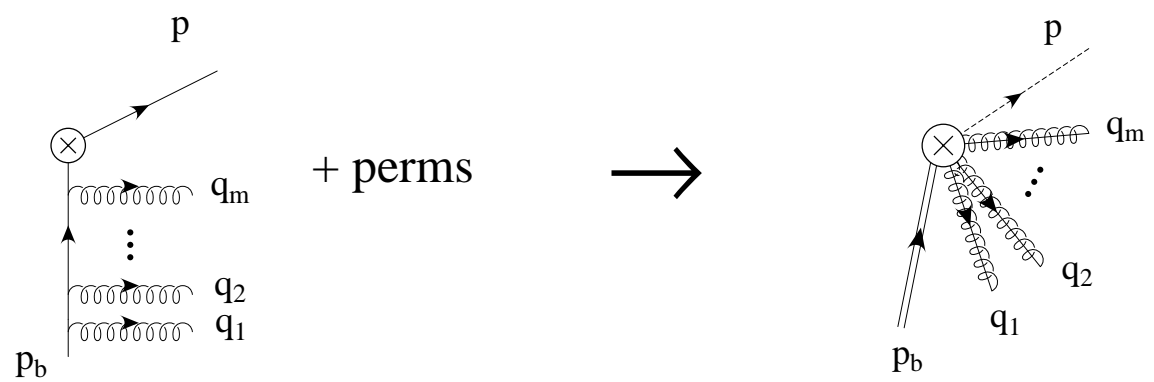

FIG. 3. Matching for the order $\lambda^{0}$ Feynman rule for the heavy to light current with $n$ collinear gluons. All permutations of crossed gluon lines are included on the left.

\section{MATCHING THE HEAVY TO LIGHT CURRENTS}

At a scale $\mu \sim Q$ the weak Hamiltonian has heavy to light semileptonic or radiative operators of the form [18]

$$
H_{e f f}=\frac{G_{F}}{\sqrt{2}} V C^{\text {full }}(\mu) J_{\text {had }} J
$$

where $V$ is the CKM factor, $J$ is a non-hadronic current, and the Wilson coefficients $C^{\text {full }}(\mu)$ have been run from the scale $\mu=m_{W}$ down to $m_{b}$. In Eq. (18), the hadronic currents are of the form $J_{\text {had }}=\bar{q} \Gamma b$ and we will consider $\Gamma=\left\{1, \gamma_{5}, \gamma_{\mu}, \gamma_{\mu} \gamma_{5}, \sigma_{\mu \nu}, \sigma_{\mu \nu} \gamma_{5}\right\}$. We choose this over-complete basis to simplify the treatment of $b \rightarrow s \gamma$. Below the scale $Q \sim \bar{n} \cdot p$ the hadronic current can be matched onto currents in the collinear-soft effective theory. This introduces a new set of Wilson coefficients $C_{i}(\mu)$. In this section the one loop matching for these new coefficients will be performed at $\mu=m_{b}$, while the running will be considered in section IV. We could equally well match at $\mu=\bar{n} \cdot p$, but the difference is irrelevant since we treat $\bar{n} \cdot p \sim m_{b}$ and do not attempt to sum logarithms of the form $\ln \left(\bar{n} \cdot p / m_{b}\right)$.

Naively, one might expect that at lowest order the effective theory hadronic current is $J_{\text {had }}^{\text {eft }}=C(\mu) \bar{\xi}_{n, p} \Gamma h_{v}$. However, since the label $\bar{n} \cdot p \sim \lambda^{0}$, the effective theory Wilson coefficient can also be a function of $\bar{n} \cdot p$. Furthermore, an arbitrary number of fields $\bar{n} \cdot A_{n, q} \sim$ $\lambda^{0}$ can be included without additional power suppression. At lowest order in $\lambda$ the most general heavy to light current in the effective theory therefore has the form

$$
\begin{aligned}
J_{\mathrm{had}}^{\mathrm{eft}}= & c_{0}(\bar{n} \cdot p, \mu) \bar{\xi}_{n, p} \Gamma h_{v}+c_{1}\left(\bar{n} \cdot p, \bar{n} \cdot q_{1}, \mu\right) \bar{\xi}_{n, p}\left(g \bar{n} \cdot A_{n, q_{1}}\right) \Gamma h_{v} \\
& +c_{2}\left(\bar{n} \cdot p, \bar{n} \cdot q_{1}, \bar{n} \cdot q_{2}, \mu\right) \bar{\xi}_{n, p}\left(g \bar{n} \cdot A_{n, q_{1}}\right)\left(g \bar{n} \cdot A_{n, q_{2}}\right) \Gamma h_{v}+\ldots,
\end{aligned}
$$

where the ellipsis stand for terms of the same order with more powers of $\bar{n} \cdot A_{n, q}$. The coefficients $c_{i}$ may also depend on the choice of $\Gamma$. At the scale $\mu=m_{b}$ the $c_{i}$ can be determined by the tree level matching calculation depicted in Fig. 3. On the left the gluons with collinear momenta kick the $b$ quark far off-shell, and integrating out these off-shell $b$ quarks gives the effective theory operator on the right. 
To perform the matching, first consider the simpler case of an Abelian gauge group. In this case calculating the full theory graph with $m$ gluons in Fig. 3, expanding in powers of $\lambda$, and putting the result over a common denominator gives

$$
c_{m}\left(\mu=m_{b}\right)=\frac{1}{m !} \prod_{i=1}^{m} \frac{1}{\bar{n} \cdot q_{i}} .
$$

The factor of $1 / m$ ! is from the presence of $m$ identical $A_{c}$ fields at the same point. Thus, we have the tree level result

$$
\left.J_{\text {had }}^{\text {eft }}\right|_{\mu=m_{b}}=\bar{\xi}_{n, p} \exp \left(\frac{g \bar{n} \cdot A_{n, q}}{\bar{n} \cdot q}\right) \Gamma h_{v} .
$$

It is not immediately clear how this result is modified for $\mu<m_{b}$ since the infinite series of operators in Eq. (19) could each run differently. However, gauge invariance relates these operators, and only the sum of terms in Eq. (21) is gauge invariant. Under a collinear gauge transformation $\alpha_{n, q}(x)$, the field $h_{v}$ is invariant since collinear gluons do not couple to heavy quarks. On the other hand, the collinear quark field transforms as $\xi_{n, p} \rightarrow e^{i \alpha(x)} \xi_{n, p}$. Thus, the operator $\bar{\xi}_{n, p} \Gamma h_{v}$ is not gauge invariant. However, it is straightforward to see that the operator in Eq. (21) is invariant, and this is done in Appendix A. It is found that

$$
\exp \left(\frac{g \bar{n} \cdot A_{n, q}}{\bar{n} \cdot q}\right) \rightarrow \exp \left(\frac{g \bar{n} \cdot A_{n, q}}{\bar{n} \cdot q}\right) \exp [i \alpha(x)],
$$

and the last exponential exactly cancels the transformation of $\bar{\xi}_{n, p}$. By gauge invariance the current therefore has to be of the form in Eq. (21) for an arbitrary scale $\mu$. It is convenient to define a field that transforms as a singlet under a collinear gauge transformation

$$
\chi_{n, P}=\exp \left(\frac{-g \bar{n} \cdot A_{n, q}}{\bar{n} \cdot q}\right) \xi_{n, p} .
$$

We will refer to $\chi_{n, P}$ as the jet field since it involves a collinear quark field plus an arbitrary number of collinear gluons moving in the $n$ direction. The relevant label for the jet field is simply the sum of labels of the particles in the jet, $P=p+\sum q_{i}$. In terms of this field the leading order effective theory current for $Q \lambda<\mu<m_{b}$ has the form

$$
J_{\mathrm{had}}^{\mathrm{eft}}=C_{i}(\mu, \bar{n} \cdot P) \bar{\chi}_{n, P} \Gamma h_{v}
$$

with a universal coefficient $C_{i}(\mu, \bar{n} \cdot P)$. The statement that the coefficient only depends on the total jet momentum $P$ is non-trivial and is discussed further in Appendix $B$.

\footnotetext{
${ }^{3}$ Note that in Ref. [6] the Feynman rule with a single collinear gluon $(m=1)$ has an additional $\not h A_{c} / m_{b}$ term which did not contribute to the results there. With the power counting in the Table 1 this term is suppressed by a power of $\lambda$.
} 


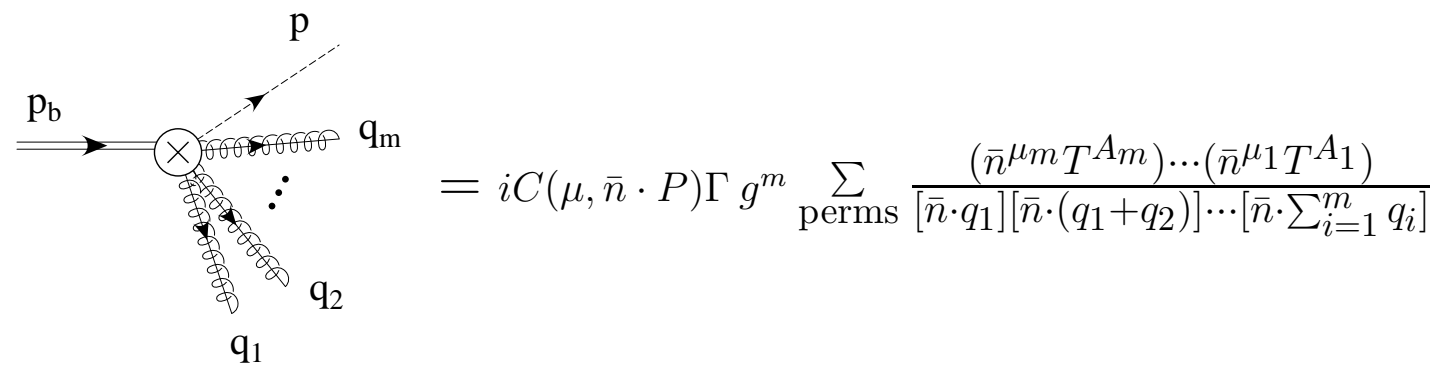

FIG. 4. Order $\lambda^{0}$ Feynman rule for the effective theory heavy to light current with $m$ collinear gluons. The sum is over permutations of $\{1, \ldots, m\}$ and the Wilson coefficient depends only on the sum of momenta in the jet, $P=p+\sum_{i=1}^{m} q_{i}$.

For a non-abelian gauge group a similar gauge invariance argument applies, however the matching in Fig. 3 is more complicated. Eq. (24) remains valid, but with a more complicated definition of the jet field. In momentum space we find

$$
\chi_{n, P}=\sum_{k} \sum_{\text {perms }} \frac{(-g)^{k}}{k !}\left(\frac{\bar{n} \cdot A_{\bar{n}, q_{1}} \cdots \bar{n} \cdot A_{\bar{n}, q_{k}}}{\left[\bar{n} \cdot q_{1}\right]\left[\bar{n} \cdot\left(q_{1}+q_{2}\right)\right] \cdots\left[\bar{n} \cdot \sum_{i=1}^{k} q_{i}\right]}\right) \xi_{n, p},
$$

where the permutation sum is over the indices $(1,2, \ldots, k)$. The Feynman rules which follow from Eqs. (24) and (25) are shown in Fig. 4. In position space the jet field takes the form of a path-ordered exponential

$$
\chi_{n}(0)=\mathrm{P} \exp \left(-i g \int_{-\infty}^{0} \mathrm{~d} s \bar{n} \cdot A^{c}\left(s \bar{n}^{\mu}\right)\right) \xi_{n}(0)
$$

where $P$ denotes path ordering along the light-like line collinear to $\bar{n} .^{4}$

In the effective theory both heavy and light quarks are described by two component spinors, so there are only four heavy to light currents at leading order in $\lambda$. We choose the linearly independent set $\left[\bar{\chi}_{n, P} h_{v}\right],\left[\bar{\chi}_{n, P} \gamma_{5} h_{v}\right]$, and $\left[\bar{\chi}_{n, P} \gamma_{\perp}^{\mu} h_{v}\right]$, where $\gamma_{\perp}^{\mu}=\gamma^{\mu}-n^{\mu} \not h / 2-\bar{n}^{\mu} \not / 2$ has only two non-zero terms. The matching of the heavy to light currents $\bar{q} \Gamma b$ onto operators in the effective theory is

$$
\begin{aligned}
\bar{q} b & \rightarrow C_{1}(\mu)\left[\bar{\chi}_{n, P} h_{v}\right], \\
\bar{q} \gamma_{5} b & \rightarrow C_{2}(\mu)\left[\bar{\chi}_{n, P} \gamma_{5} h_{v}\right], \\
\bar{q} \gamma_{\mu} b & \rightarrow C_{3}(\mu)\left[\bar{\chi}_{n, P} \gamma_{\mu}^{\perp} h_{v}\right]+\left\{C_{4}(\mu) n_{\mu}+C_{5}(\mu) v_{\mu}\right\}\left[\bar{\chi}_{n, P} h_{v}\right], \\
\bar{q} \gamma_{\mu} \gamma_{5} b & \rightarrow C_{6}(\mu) i \epsilon_{\mu \nu}^{\perp}\left[\bar{\chi}_{n, P} \gamma_{\perp}^{\nu} h_{v}\right]-\left\{C_{7}(\mu) n_{\mu}+C_{8}(\mu) v_{\mu}\right\}\left[\bar{\chi}_{n, P} \gamma_{5} h_{v}\right],
\end{aligned}
$$

\footnotetext{
${ }^{4}$ Path-ordered exponentials are also introduced to sum up the couplings of soft gluons to a collinear jet, see Ref. [19.
} 


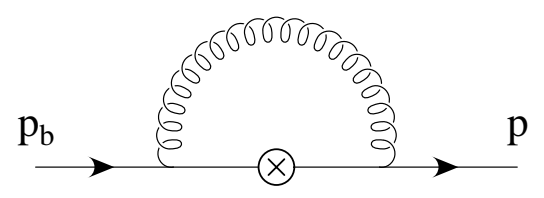

FIG. 5. Full theory one loop diagram for matching the heavy-light current (denoted by $\otimes$ ). The incoming line is a massive quark and the outgoing line is massless.

$$
\begin{aligned}
\bar{q} i \sigma_{\mu \nu} b & \rightarrow C_{9}(\mu)\left(n_{\mu} g_{\nu \lambda}-n_{\nu} g_{\mu \lambda}\right)\left[\bar{\chi}_{n, P} \gamma_{\perp}^{\lambda} h_{v}\right]+C_{10}(\mu) i \epsilon_{\mu \nu}^{\perp}\left[\bar{\chi}_{n, P} \gamma_{5} h_{v}\right] \\
& +C_{11}(\mu)\left(v_{\mu} n_{\nu}-v_{\nu} n_{\mu}\right)\left[\bar{\chi}_{n, P} h_{v}\right]+C_{12}(\mu)\left(v_{\mu} g_{\nu \lambda}-v_{\nu} g_{\mu \lambda}\right)\left[\bar{\chi}_{n, P} \gamma_{\perp}^{\lambda} h_{v}\right], \\
\bar{q} i \sigma_{\mu \nu} \gamma_{5} b & \rightarrow-\left[C_{9}(\mu)+C_{12}(\mu)\right]\left(i n_{\mu} \epsilon_{\nu \lambda}^{\perp}-i n_{\nu} \epsilon_{\mu \lambda}^{\perp}\right)\left[\bar{\chi}_{n, P} \gamma_{\perp}^{\lambda} h_{v}\right]+C_{11}(\mu) i \epsilon_{\mu \nu}^{\perp}\left[\bar{\chi}_{n, P} h_{v}\right] \\
& +C_{10}(\mu)\left(v_{\mu} n_{\nu}-v_{\nu} n_{\mu}\right)\left[\bar{\chi}_{n, P} \gamma_{5} h_{v}\right]+C_{12}(\mu)\left(i v_{\mu} \epsilon_{\nu \lambda}^{\perp}-i v_{\nu} \epsilon_{\mu \lambda}^{\perp}\right)\left[\bar{\chi}_{n, P} \gamma_{\perp}^{\lambda} h_{v}\right],
\end{aligned}
$$

where $\epsilon_{\mu \nu}^{\perp}=\epsilon_{\mu \nu \rho \sigma} v^{\rho} n^{\sigma}$ with $\epsilon_{0123}=-1$. From here on the dependence of the Wilson coefficients on $\bar{n} \cdot P$ will be suppressed. The relations in Eq. (27) are validf to all orders in $\alpha_{s}$ and leading order in $\lambda$. At tree level the matching gives

$$
C_{1,2,3,4,6,7,9,10,11}\left(m_{b}\right)=1, \quad C_{5,8,12}\left(m_{b}\right)=0 .
$$

To match these coefficients at one-loop, we calculate perturbative matrix elements in the full and effective theories. All the long distance physics is reproduced in the effective theory, and the difference between the two calculations determines the short distance Wilson coefficients. Since the Wilson coefficients are universal the matching can be performed for the simpler current $\bar{\xi}_{n, p} \Gamma h_{v}$ rather than the current $\bar{\chi}_{n, P} \Gamma h_{v}$. The calculation is most easily performed in pure dimensional regularization. The full theory matrix elements of the currents $\bar{q} \Gamma b$ between free quark states are obtained by evaluating the diagram in Fig. 5 and multiplying by the wavefunction and current renormalization factors. In $d=4-2 \epsilon$ dimensions the on-shell wavefunction renormalization constants for massive and massless quarks are

$$
Z_{b}=1+\frac{\alpha_{s} C_{F}}{4 \pi}\left(-\frac{3}{\epsilon}+3 \ln \frac{m_{b}^{2}}{\mu^{2}}-4\right), \quad Z_{q}=1
$$

and the renormalization constants for the scalar, pseudoscalar, vector, axial vector, tensor and axial tensor currents are given by

$$
Z_{S}=Z_{P}=1-\frac{3 \alpha_{s} C_{F}}{4 \pi \epsilon}, \quad Z_{V}=Z_{A}=1, \quad Z_{T}=Z_{T_{5}}=1+\frac{\alpha_{s} C_{F}}{4 \pi \epsilon}
$$

\footnotetext{
${ }^{5}$ An exception is the relation between the coefficients for $\bar{q} i \sigma_{\mu \nu} b$ and $\bar{q} i \sigma_{\mu \nu} \gamma_{5} b$ which can change depending on how $\gamma_{5}$ is treated in $d$ dimensions.
} 
The ultraviolet divergences in the $Z$ 's cancel the ultraviolet divergences in the diagram in Fig. 5, hence all remaining $1 / \epsilon$ divergences are of infrared nature. The $b$ quark and light quark are taken to have momenta $p_{b}$ and $p$ respectively, and we define $q=p_{b}-p$. Letting $\gamma_{5}$ anticommute in $d$ dimensions (the NDR scheme), the final result for the matrix elements in the full theory is

$$
\begin{aligned}
\left\langle q\left|\bar{q}\left\{1, \gamma_{5}\right\} b\right| b\right\rangle= & 1-\frac{\alpha_{s} C_{F}}{4 \pi}\left[\frac{1}{\epsilon^{2}}+\frac{5}{2 \epsilon}+\frac{\ln \left(\frac{\mu^{2}}{m_{b}^{2}}\right)}{\epsilon}-\frac{2 \ln \left(1-\hat{q}^{2}\right)}{\epsilon}+\frac{1}{2} \ln ^{2}\left(\frac{\mu^{2}}{m_{b}^{2}}\right)\right. \\
& -\frac{1}{2} \ln \left(\frac{\mu^{2}}{m_{b}^{2}}\right)-2 \ln \left(1-\hat{q}^{2}\right) \ln \left(\frac{\mu^{2}}{m_{b}^{2}}\right)+2 \ln ^{2}\left(1-\hat{q}^{2}\right) \\
& \left.\left.-\frac{2 \ln \left(1-\hat{q}^{2}\right)}{\hat{q}^{2}}+2 \operatorname{Li}_{2}\left(\hat{q}^{2}\right)+\frac{\pi^{2}}{12}\right]\right\} \bar{u}\left\{1, \gamma_{5}\right\} u, \\
\left\langle q\left|\bar{q}\left\{1, \gamma_{5}\right\} \gamma^{\mu} b\right| b\right\rangle= & \left\{1-\frac{\alpha_{s} C_{F}}{4 \pi}\left[\frac{1}{\epsilon^{2}}+\frac{5}{2 \epsilon}+\frac{\ln \left(\frac{\mu^{2}}{m_{b}^{2}}\right)}{\epsilon}-\frac{2 \ln \left(1-\hat{q}^{2}\right)}{\epsilon}+\frac{1}{2} \ln ^{2}\left(\frac{\mu^{2}}{m_{b}^{2}}\right)\right.\right. \\
& +\frac{5}{2} \ln \left(\frac{\mu^{2}}{m_{b}^{2}}\right)-2 \ln \left(1-\hat{q}^{2}\right) \ln \left(\frac{\mu^{2}}{m_{b}^{2}}\right)+2 \ln ^{2}\left(1-\hat{q}^{2}\right) \\
& \left.\left.+\ln \left(1-\hat{q}^{2}\right)\left(\frac{1}{\hat{q}^{2}}-3\right)+2 \operatorname{Li}_{2}\left(\hat{q}^{2}\right)+\frac{\pi^{2}}{12}+6\right]\right\} \bar{u}\left\{1, \gamma_{5}\right\} \gamma^{\mu} u \\
& +\frac{\alpha_{s} C_{F}}{4 \pi}\left[\frac{4}{\hat{q}^{2}} \ln \left(1-\hat{q}^{2}\right)-\frac{2}{\hat{q}^{2}}-\frac{2}{\hat{q}^{4}} \ln \left(1-\hat{q}^{2}\right)\right] \hat{p}^{\mu} \bar{u}\left\{1, \gamma_{5}\right\} u \\
& +\frac{\alpha_{s} C_{F}}{4 \pi}\left[\frac{2}{\hat{q}^{2}}-\frac{2}{\hat{q}^{2}} \ln \left(1-\hat{q}^{2}\right)+\frac{2}{\hat{q}^{4}} \ln \left(1-\hat{q}^{2}\right)\right] \hat{p}_{b}^{\mu} \bar{u}\left\{1, \gamma_{5}\right\} u, \\
\left\langle q\left|\bar{q}\left\{1, \gamma_{5}\right\} i \sigma^{\mu \nu} b\right| b\right\rangle= & {\left[1-\frac{\alpha_{s} C_{F}}{4 \pi}\left[\frac{1}{\epsilon^{2}}+\frac{5}{2 \epsilon}+\frac{\ln \left(\frac{\mu^{2}}{m_{b}^{2}}\right)}{\epsilon}-\frac{2 \ln \left(1-\hat{q}^{2}\right)}{\epsilon}+\frac{1}{2} \ln ^{2}\left(\frac{\mu^{2}}{m_{b}^{2}}\right)\right.\right.} \\
& +\frac{7}{2} \ln \left(\frac{\mu^{2}}{m_{b}^{2}}\right)-2 \ln \left(1-\hat{q}^{2}\right) \ln \left(\frac{\mu^{2}}{m_{b}^{2}}\right)+2 \ln ^{2}\left(1-\hat{q}^{2}\right) \\
& \left.\left.+2 \ln \left(1-\hat{q}^{2}\right)\left(\frac{1}{\hat{q}^{2}}-2\right)+2 \operatorname{Li}_{2}\left(\hat{q}^{2}\right)+\frac{\pi^{2}}{12}+6\right]\right\} \bar{u}\left\{1, \gamma_{5}\right\} i \sigma^{\mu \nu} u \\
& +\frac{\alpha_{s} C_{F}}{4 \pi}\left[\frac{4}{\hat{q}^{2}} \ln \left(1-\hat{q}^{2}\right)\right] \bar{u}\left\{1, \gamma_{5}\right\}\left(\hat{p}^{\mu} \gamma^{\nu}-\hat{p}^{\nu} \gamma^{\mu}\right) u,
\end{aligned}
$$

where the hat denotes momenta normalized with respect to $m_{b}$, so $\hat{q}=q / m_{b}$. This full theory result can be expanded in $\lambda$ by noting that

$$
\hat{q}^{2}=1-\bar{n} \cdot \hat{p}+\mathcal{O}\left(\lambda^{2}\right),
$$

and that at lowest order we can expand the full theory spinors using Eqs. (27) and (28).

For the effective theory in pure dimensional regularization the final collinear quark is taken on-shell. For momentum labels $\left(\bar{n} \cdot p, p_{\perp}\right)$ this corresponds to choosing this quarks residual momentum $k$ such that $\bar{n} \cdot p n \cdot k+p_{\perp}^{2}=0$. In this case all graphs in the effective 
theory are proportional to $1 / \epsilon_{\mathrm{UV}}-1 / \epsilon_{\mathrm{IR}}=0$. The ultraviolet divergences are canceled by effective theory counterterms, and all infrared divergences cancel in the difference between the full and effective theories. Thus, from Eq. (31) the Wilson coefficients at the scale $\mu=m_{b}$ are

$$
\begin{aligned}
& C_{1,2}\left(m_{b}\right)=1-\frac{\alpha_{s}\left(m_{b}\right) C_{F}}{4 \pi}\left\{2 \ln ^{2}(\bar{n} \cdot \hat{P})+2 \operatorname{Li}_{2}(1-\bar{n} \cdot \hat{P})-\frac{2 \ln (\bar{n} \cdot \hat{P})}{1-\bar{n} \cdot \hat{P}}+\frac{\pi^{2}}{12}\right\}, \\
& C_{3,6}\left(m_{b}\right)=1-\frac{\alpha_{s}\left(m_{b}\right) C_{F}}{4 \pi}\left\{2 \ln ^{2}(\bar{n} \cdot \hat{P})+2 \operatorname{Li}_{2}(1-\bar{n} \cdot \hat{P})+\ln (\bar{n} \cdot \hat{P})\left(\frac{3 \bar{n} \cdot \hat{P}-2}{1-\bar{n} \cdot \hat{P}}\right)+\frac{\pi^{2}}{12}+6\right\}, \\
& C_{4,7}\left(m_{b}\right)=1-\frac{\alpha_{s}\left(m_{b}\right) C_{F}}{4 \pi}\left\{2 \ln ^{2}(\bar{n} \cdot \hat{P})+2 \operatorname{Li}_{2}(1-\bar{n} \cdot \hat{P})-\ln (\bar{n} \cdot \hat{P})\left[\frac{2-4 \bar{n} \cdot \hat{P}+(\bar{n} \cdot \hat{P})^{2}}{(1-\bar{n} \cdot \hat{P})^{2}}\right]\right. \\
& \left.+\frac{\bar{n} \cdot \hat{P}}{1-\bar{n} \cdot \hat{P}}+\frac{\pi^{2}}{12}+6\right\} \\
& C_{5,8}\left(m_{b}\right)=\frac{\alpha_{s}\left(m_{b}\right) C_{F}}{4 \pi}\left\{\frac{2}{(1-\bar{n} \cdot \hat{P})}+\frac{2 \bar{n} \cdot \hat{P} \ln (\bar{n} \cdot \hat{P})}{(1-\bar{n} \cdot \hat{P})^{2}}\right\} \text {, } \\
& C_{9}\left(m_{b}\right)=1-\frac{\alpha_{s}\left(m_{b}\right) C_{F}}{4 \pi}\left\{2 \ln ^{2}(\bar{n} \cdot \hat{P})+2 \operatorname{Li}_{2}(1-\bar{n} \cdot \hat{P})-2 \ln (\bar{n} \cdot \hat{P})+\frac{\pi^{2}}{12}+6\right\}, \\
& C_{10,11}\left(m_{b}\right)=1-\frac{\alpha_{s}\left(m_{b}\right) C_{F}}{4 \pi}\left[2 \ln ^{2}(\bar{n} \cdot \hat{P})+2 \operatorname{Li}_{2}(1-\bar{n} \cdot \hat{P})+\ln (\bar{n} \cdot \hat{P})\left(\frac{4 \bar{n} \cdot \hat{P}-2}{1-\bar{n} \cdot \hat{P}}\right)+\frac{\pi^{2}}{12}+6\right] \text {, } \\
& C_{12}\left(m_{b}\right)=0 .
\end{aligned}
$$

For the operator $\bar{\xi}_{n, p} \Gamma h_{v}$ there is only one particle in the jet, so in that case $P=p$. In NDR the relations amongst Wilson coefficients, $C_{1}=C_{2}, C_{3}=C_{6}, C_{4}=C_{7}, C_{5}=C_{8}, C_{10}=C_{11}$, and $C_{12}=0$ hold true to all orders in perturbation theory for a massless light quark. This is because the transformation, $q \rightarrow \gamma_{5} q$ is a symmetry of massless QCD and the U(1) helicity symmetry of Eq. (12) allows $\chi_{n, p} \rightarrow \gamma_{5} \chi_{n, p}$, and these transformations relate currents with and without $\gamma_{5}$.

\section{RENORMALIZATION GROUP EVOLUTION}

In this section we calculate the running of the Wilson coefficients in the effective theory. The coefficients mix into themselves and satisfy a renormalization group equation of the form

$$
\mu \frac{d}{d \mu} C(\mu)=\gamma(\mu) C(\mu) .
$$

The fact that Eq. (34) is homogeneous reproduces the exponentiation of Sudakov logarithms. In this case it is natural to solve the renormalization group equations for the quantity 


\begin{tabular}{lc|ccc}
\hline \hline \multicolumn{1}{c|}{ series in $\ln C(\mu)$} & one-loop & two-loops & three-loops \\
\hline $\mathrm{LL}$ & $\alpha_{s}^{n} \ln ^{n+1}$ & $1 / \epsilon^{2}$ & - & - \\
$\mathrm{NLL}$ & $\alpha_{s}^{n} \ln ^{n}$ & $1 / \epsilon$ & $1 / \epsilon^{2}$ & - \\
$\mathrm{NNLL}$ & $\alpha_{s}^{n} \ln ^{n-1}$ & matching & $1 / \epsilon$ & $1 / \epsilon^{2}$ \\
\hline \hline
\end{tabular}

TABLE II. Coefficients in the effective theory loop graphs which we anticipate are needed to predict the series of Sudakov logarithms in $\ln C(\mu)$.

$\ln C(\mu)$. The leading and subleading series of logarithms are determined by the coefficients summarized in Table. III. From the Table one can see that for coefficients with tree level matching, the one-loop matching in section $\Pi 1]$ is not needed until NNLL order.

In section III it was shown that the coefficient of the effective theory current $\bar{\chi}_{n, P} \Gamma h_{v}$ is the same as the current $\bar{\xi}_{n, p} \Gamma h_{v}$, so only the renormalization of the simpler $\bar{\xi}_{n, p} \Gamma h_{v}$ current needs to be considered. At one-loop the effective theory diagrams are shown in Fig. 6. To distinguish UV and IR divergences we choose the collinear quark momentum $p=\tilde{p}+k$ with label $\tilde{p}=\left(\bar{n} \cdot p, 0, p_{\perp}\right)$ and zero residual momentum, $k=0$. In this case $p^{2}=p_{\perp}^{2} \neq 0$ and this off-shellness regulates IR divergences in the diagrams. We will use Feynman gauge. The soft diagrams in Fig. 6 give

$$
\begin{aligned}
& \text { Fig. } 6 \mathrm{a}=i \bar{\xi}_{n, p} \Gamma h_{v} \frac{C_{F} \alpha_{s}(\mu) C(\mu)}{4 \pi}\left[-\frac{1}{\epsilon^{2}}-\frac{2}{\epsilon} \ln \left(\frac{\mu \bar{n} \cdot p}{-p_{\perp}^{2}-i \epsilon}\right)-2 \ln ^{2}\left(\frac{\mu \bar{n} \cdot p}{-p_{\perp}^{2}-i \epsilon}\right)-\frac{3 \pi^{2}}{4}\right], \\
& \text { Fig. } 6 \mathrm{~b}=i v \cdot k \frac{\alpha_{s}(\mu) C_{F}}{4 \pi}\left[-\frac{2}{\epsilon}-4-4 \ln \left(\frac{\mu}{-2 v \cdot k-i \epsilon}\right)\right],
\end{aligned}
$$

where $k$ is a residual momentum in the heavy quark wavefunction diagram. The order $\lambda^{0}$ soft wavefunction renormalization of the collinear quark is not shown since in Feynman gauge it is proportional to $n^{2}=0$. Evaluating the diagrams with a collinear gluon in Fig. 6 gives

$$
\begin{aligned}
\text { Fig. } 6 \mathrm{c}= & i \bar{\xi}_{n, p} \Gamma h_{v} \frac{C_{F} \alpha_{s}(\mu) C(\mu)}{4 \pi}\left[\frac{2}{\epsilon^{2}}+\frac{2}{\epsilon}+\frac{2}{\epsilon} \ln \left(\frac{\mu^{2}}{-p_{\perp}^{2}-i \epsilon}\right)+\ln ^{2}\left(\frac{\mu^{2}}{-p_{\perp}^{2}-i \epsilon}\right)\right. \\
& \left.+2 \ln \left(\frac{\mu^{2}}{-p_{\perp}^{2}-i \epsilon}\right)+4-\frac{\pi^{2}}{6}\right], \\
\text { Fig. 6d } \mathrm{d}= & \frac{i \hbar h}{2} \frac{p_{\perp}^{2}}{\bar{n} \cdot p} \frac{\alpha_{s}(\mu) C_{F}}{4 \pi}\left[\frac{1}{\epsilon}+1+\ln \left(\frac{\mu^{2}}{-p_{\perp}^{2}-i \epsilon}\right)\right],
\end{aligned}
$$

Fig. 6e, $\mathrm{f}=0$.

The graph in Fig. 6d was calculated explicitly in section II. 
a)

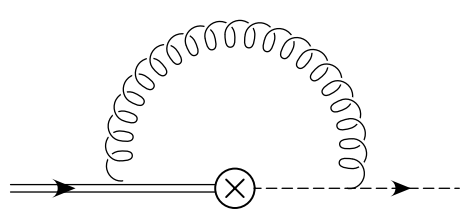

b)

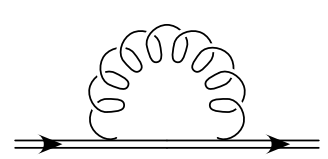

c)

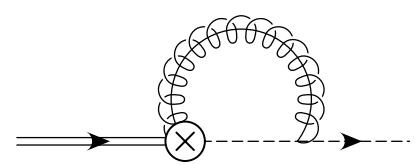

d)

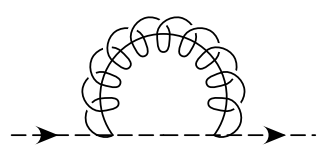

e)

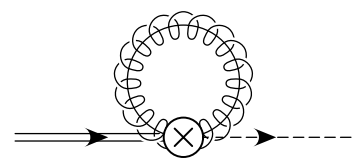

f)

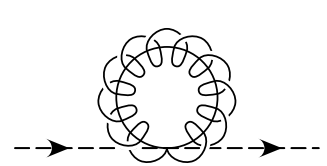

FIG. 6. Order $\lambda^{0}$ effective theory diagrams for the heavy to light current at one loop.

From Eq. (35) we see that the logarithms in diagrams with collinear gluons are small at a scale $\mu \sim \sqrt{p_{\perp}^{2}} \sim Q \lambda$. For the graphs with soft gluons the logarithms are small at a different scale $\mu \sim p_{\perp}^{2} /(\bar{n} \cdot p) \sim Q \lambda^{2}$. Running the collinear-soft theory from $\mu=Q$ to $\mu=Q \lambda$ therefore sums all logarithms originating from collinear effects and part of the logarithms from soft exchange. At $\mu=Q \lambda$ collinear gluons may be integrated out and one matches onto a theory containing only soft degrees of freedom. The running in this soft theory includes the remaining logarithms from soft exchange, which would need to be taken into account to sum all Sudakov logarithms.

To run between $Q$ and $Q \lambda$ we add up the ultraviolet divergences in the soft and collinear diagrams in Eqs. (35) and (36). This gives the counterterm in the effective theory

$$
Z_{i}=1+\frac{\alpha_{s}(\mu) C_{F}}{4 \pi}\left[\frac{1}{\epsilon^{2}}+\frac{2}{\epsilon} \ln \left(\frac{\mu}{\bar{n} \cdot P}\right)+\frac{5}{2 \epsilon}\right] .
$$

For $b \rightarrow s \gamma, \bar{n} \cdot P=m_{b}$ and Eq. (37) agrees with Ref. [6]. Since $\mu>Q \lambda$ the counterterm can depend on the label $\bar{n} \cdot P \sim Q$, but does not depend on $P_{\perp} \sim Q \lambda$. $Z_{i}$ could also have been calculated directly from the matching result in Eq. (31). Since the effective theory reproduces all the infrared divergences in the full theory, the effective theory UV divergences are simply the negative of the full theory IR divergences when pure dimensional regularization is used. This alternative approach also gives Eq. (37).

In the effective theory the current $\bar{\xi}_{n, p} \Gamma h_{v}$ factors out of the diagrams in Fig. 6 so it is obvious that $Z_{i}$ is independent of the spin structure of the current. Thus, all the coefficients satisfy the same renormalization group equation (RGE)

$$
\mu \frac{d}{d \mu} C_{i}(\mu)=\gamma(\mu) C_{i}(\mu)
$$

The LO anomalous dimension is determined by the $\ln (\mu) / \epsilon$ term in Eq. (37) (whose coefficient is determined by the $1 / \epsilon^{2}$ term). The NLO anomalous dimension has a contribution from the $1 / \epsilon$ terms in Eq. (37), as well as a contribution from the $\ln (\mu) / \epsilon$ term in the two loop $Z_{i}$ counterterm: 


$$
\begin{aligned}
\gamma_{L O} & =-\frac{\alpha_{s}(\mu) C_{F}}{\pi} \ln \left(\frac{\mu}{\bar{n} \cdot P}\right), \\
\gamma_{N L O} & =-\frac{5 \alpha_{s}(\mu) C_{F}}{4 \pi}-2 C_{F} B \frac{\alpha_{s}^{2}(\mu)}{(2 \pi)^{2}} \ln \left(\frac{\mu}{\bar{n} \cdot P}\right) .
\end{aligned}
$$

We have introduced the notation $B$ for the two loop coefficient which has not yet been computed with the effective theory. From the results in Ref. [9] we are led to expect that $B=C_{A}\left(67 / 18-\pi^{2} / 6\right)-5 n_{f} / 9$.

Since we wish to run down from $\mu=m_{b}$ and $\bar{n} \cdot P \sim m_{b}$ it is convenient to introduce the scale $m_{b}$ into the anomalous dimensions. Writing $\ln (\mu / \bar{n} \cdot p)=\ln \left(\mu / m_{b}\right)-\ln (\bar{n} \cdot \hat{P})$ and noting that the second logarithm is not large, Eq. (39) can be written as

$$
\begin{aligned}
\gamma_{L O} & =-\frac{\alpha_{s}(\mu) C_{F}}{\pi} \ln \left(\frac{\mu}{m_{b}}\right), \\
\gamma_{N L O} & =-\frac{\alpha_{s}(\mu) C_{F}}{2 \pi}\left[\frac{5}{2}-2 \ln (\bar{n} \cdot \hat{P})\right]-2 C_{F} B \frac{\alpha_{s}^{2}(\mu)}{(2 \pi)^{2}} \ln \left(\frac{\mu}{m_{b}}\right) .
\end{aligned}
$$

Using the one-loop running for $\alpha_{s}(\mu)$ the LO solution of the RGE is

$$
\ln \left[\frac{C_{i}(\mu)}{C_{i}\left(m_{b}\right)}\right]=\frac{f_{0}(z)}{\alpha_{s}\left(m_{b}\right)}=-\frac{4 \pi C_{F}}{\beta_{0}^{2} \alpha_{s}\left(m_{b}\right)}\left[\frac{1}{z}-1+\ln z\right],
$$

where $\beta_{0}=11 / 3 C_{A}-2 / 3 n_{f}$ and

$$
z=\frac{\alpha_{s}(\mu)}{\alpha_{s}\left(m_{b}\right)}=\frac{2 \pi}{2 \pi+\beta_{0} \alpha_{s}\left(m_{b}\right) \ln \left(\mu / m_{b}\right)} .
$$

Eq. (41) sums the LL series of Sudakov logarithms between $Q$ and $Q \lambda$. At NLL order we include the $\gamma_{N L O}$ term in the anomalous dimension and the two-loop running of $\alpha_{s}(\mu)$ in $\gamma_{L O}$ and find the following correction to Eq. (41) :

$$
\begin{aligned}
\left.\ln \left[\frac{C_{i}(\mu)}{C_{i}\left(m_{b}\right)}\right]\right|_{N L O}=f_{1}(z, \bar{n} \cdot \hat{P})= & -\frac{C_{F} \beta_{1}}{\beta_{0}^{3}}\left[1-z+z \ln z-\frac{1}{2} \ln ^{2} z\right] \\
& +\frac{C_{F}}{\beta_{0}}\left[\frac{5}{2}-2 \ln (\bar{n} \cdot \hat{P})\right] \ln z-\frac{2 C_{F} B}{\beta_{0}^{2}}[z-1-\ln z] .
\end{aligned}
$$

Here $\beta_{1}=34 C_{A}^{2} / 3-10 C_{A} n_{f} / 3-2 C_{F} n_{f}$, and $z$ is still given by Eq. (42). It is easy to see that the result in Eq. (43) is suppressed by an extra $\alpha_{s}(m)$ relative to the result in Eq. (41). Also it is clear that to systematically sum the next-to-leading log series the two loop coefficient $B$ is required.

Combining Eqs. (41) and (43) the final result at a scale $\mu \sim Q \lambda$ is

$$
C_{i}(\mu)=C_{i}\left(m_{b}\right) \exp \left[\frac{f_{0}(z)}{\alpha_{s}\left(m_{b}\right)}+f_{1}(z)\right] .
$$

For $i=\{1,2,3,4,6,7,9,10,11\}$ the matching starts at tree level and from Table $\mathbb{\Pi}$ we see that for the LL and NLL solutions the value $C_{i}\left(m_{b}\right)=1$ should be used in Eq. (44). The coefficients $C_{5,8,12}\left(m_{b}\right)$ are zero at tree level, and inserting their one-loop matching values from Eq. (33) into Eq. (44) gives their LL and NLL series of logarithms. 


\section{APPLICATIONS}

\section{A. Inclusive decays}

It is well known that the OPE for heavy-to-light decays converges only for sufficiently inclusive variables. If the available phase space is restricted such that only a few resonances contribute to the decay the assumption of local duality no longer holds and the OPE fails. If, however, phase space is restricted such that highly energetic jets with small invariant mass dominate the decay, only a subset of terms in the OPE are enhanced. It is possible to resum this subset of terms into a universal structure function [20]. In the same region of phase space large Sudakov logarithms spoil the perturbative expansion and thus have to be summed as well. This summation was carried out for the endpoint of the leptonic energy spectrum in inclusive $B \rightarrow X_{u} \ell \bar{\nu}$ decays and the endpoint of the photonic energy spectrum in inclusive $B \rightarrow X_{s} \gamma$ decays [9,21] using perturbative factorization [19. In a subsequent work [10 endpoint logarithms in the hadronic mass spectrum of $B \rightarrow X_{u} \ell \bar{\nu}$ decays were summed within the same approach. In [6] it was shown that the result for $B \rightarrow X_{s} \gamma$ can be reproduced using the effective field theory.

In this section we consider $B \rightarrow X_{s} \gamma$ and $B \rightarrow X_{u} \ell \bar{\nu}$ decays at the endpoint of the photonic energy and hadronic invariant mass spectrum, respectively. We define kinematic variables

$$
s_{0}=\frac{p_{u}^{2}}{m_{b}^{2}}, \quad h=\frac{2 v \cdot p_{u}}{m_{b}},
$$

for $B \rightarrow X_{u} \ell \bar{\nu}$ decays where $p_{u}$ is the momentum of the $u$ quark. For $B \rightarrow X_{s} \gamma$ decays we define

$$
x=\frac{2 v \cdot q}{m_{b}},
$$

where $q$ is the photon momentum. The endpoint regions mentioned above correspond to $1-x \sim s_{0} / h \sim \Lambda_{Q C D} / m_{b}$. Thus, the invariant mass of the light jet is of order $\sqrt{m_{b} \Lambda_{Q C D}}$, and the power counting parameter satisfies $\lambda^{2} \sim \Lambda_{Q C D} / m_{b}$. At tree level, integrating out collinear modes by performing an OPE in the collinear-soft theory and matching onto soft operators gives

$$
\begin{aligned}
\frac{d \widehat{\Gamma}_{s}}{d x} & \equiv \frac{1}{\Gamma_{s}^{(0)}} \frac{d \Gamma_{s}}{d x}=\langle B|O(x)| B\rangle \\
\frac{d^{2} \widehat{\Gamma}_{u}}{d z d h} & \equiv \frac{1}{\Gamma_{u}^{(0)}} \frac{d^{2} \Gamma_{u}}{d z d h}=2 h^{2}(3-2 h)\langle B|O(z)| B\rangle,
\end{aligned}
$$

where $z=1-s_{0} / h$. Here we defined the tree level decay rates in the parton model 


$$
\Gamma_{u}^{(0)}=\frac{G_{F}^{2}}{192 \pi^{3}}\left|V_{u b}\right|^{2} m_{b}^{5}, \quad \Gamma_{s}^{(0)}=\frac{G_{F}^{2}}{32 \pi^{4}}\left|V_{t s}^{*} V_{t b}\right|^{2} \alpha_{\mathrm{em}}\left[C_{7}^{\text {full }}\right]^{2} m_{b}^{5},
$$

where $C_{7}^{\text {full }}$ is the Wilson coefficient of the weak operator mediating the $b \rightarrow s$ radiative transition [18] and we neglect contributions from operators other than $O_{7}^{\text {full }}$. The operator appearing in Eq. (47) is defined as [20]

$$
O(y)=\bar{h}_{v} \delta\left(i \hat{D}_{+}+1-y\right) h_{v}
$$

where the covariant derivative $\hat{D}_{+}=D_{+} / m_{b}$ includes only soft gluons. The matrix element of this operator between $B$-meson states is the light-cone structure function of the $B$ meson. At higher orders in perturbation theory the differential decay rates can be expressed as convolutions of short distance coefficients with the operator $O(y)$. Defining moments of the decay rates

$$
\begin{aligned}
\frac{d \widehat{\Gamma}_{u}(N)}{d h} & =\frac{1}{\Gamma_{u}^{(0)}} \int_{0}^{1} d z z^{N-1} \frac{d^{2} \Gamma_{u}}{d z d h}, \\
\widehat{\Gamma}_{s}(N) & =\frac{1}{\Gamma_{s}^{(0)}} \int_{0}^{1} d x x^{N-1} \frac{d \Gamma_{s}}{d x},
\end{aligned}
$$

undoes the convolution, and makes comparison to existing results in the literature straightforward. Let $\mu_{0} \sim Q \lambda$ be the scale where collinear modes are integrated out. At this scale the moments of the decay rates are

$$
\begin{aligned}
\frac{d \widehat{\Gamma}_{u}(N)}{d h} & =2 h^{2}(3-2 h) C\left(\mu_{0}, h m_{b}\right)^{2}\left\langle O\left(N ; \mu_{0}\right)\right\rangle, \\
\widehat{\Gamma}_{s}(N) & =C\left(\mu_{0}, m_{b}\right)^{2}\left\langle O\left(N ; \mu_{0}\right)\right\rangle,
\end{aligned}
$$

where the operator $O\left(N ; \mu_{0}\right)$ is defined as

$$
O\left(N ; \mu_{0}\right)=\int_{0}^{1} d y y^{N-1} O\left(y ; \mu_{0}\right) .
$$

Various coefficients $C_{i}(\mu, \bar{n} \cdot p)$ can contribute to the decay rates in Eq. (51). However, at NLL order we only need the tree level matching at $\mu=m_{b}$ in Eq. (28). Furthermore, at the scale $\mu_{0}=m_{b} / \sqrt{N}$ large logarithms are not introduced when matching onto the operator $O\left(N, \mu_{0}\right)$ [6]. At this scale to NLL order we therefore only need tree level matching onto the operator $O\left(N, m_{b} / \sqrt{N}\right)$. Since between $\mu=m_{b}$ and $\mu=m_{b} / \sqrt{N}$ all coefficients $C_{i}(\mu, \bar{n} \cdot p)$ have universal running the result can be written in terms of a single coefficient $C(\mu, \bar{n} \cdot p)$, where $C\left(m_{b}, \bar{n} \cdot p\right)=1$ and runs according to Eq. (44).

The running of $C(\mu, \bar{n} \cdot p)$ does not reproduce the full set of Sudakov logarithms because at $\mu=m_{b} / \sqrt{N}$ there are additional large logarithms in the matrix element of $O(N, \mu)$. It 
has been shown that these additional logarithms arise from purely soft gluons and can be summed by running from $\mu=m_{b} / \sqrt{N}$ to $\mu=m_{b} / N$ [6]. However, taking the ratio of the decay rates in Eq. (50) these matrix elements cancel:

$$
\frac{1}{\widehat{\Gamma}_{s}(N)} \frac{d \widehat{\Gamma}_{u}(N)}{d h}=2 h^{2}(3-2 h)\left[\frac{C\left(\mu_{0}, m_{b} h\right)}{C\left(\mu_{0}, m_{b}\right)}\right]^{2} .
$$

Thus, all the Sudakov logarithms in the ratio of rates are calculable from the running of the Wilson coefficients in the collinear soft theory. Using Eq. (44) this leads to

$$
\frac{1}{\widehat{\Gamma}_{s}(N)} \frac{d \widehat{\Gamma}_{u}(N)}{d h}=2 h^{2}(3-2 h) \exp \left(-\frac{4 C_{F}}{\beta_{0}} \ln (h) \ln (z)\right),
$$

where $z(\mu)=\alpha_{s}(\mu) / \alpha_{s}\left(m_{b}\right)$ is evaluated at $\mu=m_{b} / \sqrt{N}$. This result agrees with Ref. [10].

\section{B. Exclusive decays}

As another application of the results obtained in sections II and III, we investigate exclusive heavy to light decays. The nonperturbative physics of such decays is given in terms of form factors. For $B$ decays to pseudoscalar and vector mesons they are conventionally defined as

$$
\begin{aligned}
\left\langle P(p)\left|\bar{q} \gamma^{\mu} b\right| \bar{B}\left(p_{b}\right)\right\rangle & =f_{+}\left(q^{2}\right)\left[p_{b}^{\mu}+p^{\mu}-\frac{m_{B}^{2}-m_{P}^{2}}{q^{2}} q^{\mu}\right]+f_{0}\left(q^{2}\right) \frac{m_{B}^{2}-m_{P}^{2}}{q^{2}} q^{\mu}, \\
\left\langle P(p)\left|\bar{q} i \sigma^{\mu \nu} q_{\nu} b\right| \bar{B}\left(p_{b}\right)\right\rangle & =-\frac{f_{T}\left(q^{2}\right)}{m_{B}+m_{P}}\left[q^{2}\left(p_{b}^{\mu}+p^{\mu}\right)-\left(m_{B}^{2}-m_{P}^{2}\right) q^{\mu}\right], \\
\left\langle V\left(p, \epsilon^{*}\right)\left|\bar{q} \gamma^{\mu} b\right| \bar{B}\left(p_{b}\right)\right\rangle & =\frac{2 V\left(q^{2}\right)}{m_{B}+m_{V}} i \epsilon^{\mu \nu \rho \sigma} \epsilon_{\nu}^{*}\left(p_{b}\right)_{\rho} p_{\sigma}, \\
\left\langle V\left(p, \epsilon^{*}\right)\left|\bar{q} \gamma^{\mu} \gamma_{5} b\right| \bar{B}\left(p_{b}\right)\right\rangle & =2 m_{V} A_{0}\left(q^{2}\right) \frac{\epsilon^{*} \cdot q}{q^{2}} q^{\mu}+\left(m_{B}+m_{V}\right) A_{1}\left(q^{2}\right)\left[\epsilon^{* \mu}-\frac{\epsilon^{*} \cdot q}{q^{2}} q^{\mu}\right] \\
-A_{2}\left(q^{2}\right) & \frac{\epsilon^{*} \cdot q}{m_{B}+m_{V}}\left[p_{b}^{\mu}+p^{\mu}-\frac{m_{B}^{2}-m_{V}^{2}}{q^{2}} q^{\mu}\right], \\
\left\langle V\left(p, \epsilon^{*}\right)\left|\bar{q} i \sigma^{\mu \nu} q_{\nu} b\right| \bar{B}\left(p_{b}\right)\right\rangle & =-2 T_{1}\left(q^{2}\right) i \epsilon^{\mu \nu \rho \sigma} \epsilon_{\nu}^{*}\left(p_{b}\right)_{\rho} p_{\sigma}, \\
\left\langle V\left(p, \epsilon^{*}\right)\left|\bar{q} i \sigma^{\mu \nu} \gamma_{5} q_{\nu} b\right| \bar{B}\left(p_{b}\right)\right\rangle & =T_{2}\left(q^{2}\right)\left[\left(m_{B}^{2}-m_{V}^{2}\right) \epsilon^{* \mu}-\left(\epsilon^{*} \cdot q\right)\left(p_{b}^{\mu}+p^{\mu}\right)\right] \\
+T_{3}\left(q^{2}\right) & \left(\epsilon^{*} \cdot q\right)\left[q^{\mu}-\frac{q^{2}}{m_{B}^{2}-m_{V}^{2}}\left(p_{b}^{\mu}+p^{\mu}\right)\right],
\end{aligned}
$$

where $q=p_{b}-p$. For decays in which the final light meson has large energy we can use the effective theory to gain additional information on these form factors. Using Eq. (27) the matrix elements in the full theory are given by matrix elements in the effective theory 


$$
\left.\langle M|\bar{q} \Gamma b| B\rangle \rightarrow \sum_{i} C_{i}(\mu)\left\langle M_{n, P}\left|\bar{\chi}_{n, P} \Gamma_{i} h_{v}\right| H_{v}\right\rangle\right|_{\mu}+\Delta F_{\Gamma} .
$$

Here $M=P, V$ correspond to the light pseudoscalar and vector meson states in the full theory, and $M_{n, P}$ and $H_{v}$ are the states of the light and the heavy mesons in the effective theory, respectively. The first term in Eq. (56) is the soft contribution, while the second term indicates the so-called hard contributions [22,13]. For the soft form factor the offshellness of the light quark $p_{q}^{2}=2 E k_{+}$where $k_{+} \sim \Lambda_{\mathrm{QCD}}$, thus $\lambda^{2} \sim \Lambda_{\mathrm{QCD}} / m_{b}$, just as for the inclusive decays. The $\Delta F_{\Gamma}$ term in Eq. (56) involves interactions where a collinear gluon is exchanged with the spectator in the $B$ meson. In Ref. [13] it was argued that these spectator effects are the same order in $\lambda$ and $1 / m_{b}$ as the soft contributions, but can be regarded as being suppressed by a power of $\alpha_{s}\left(\sqrt{m_{b} \Lambda_{\mathrm{QCD}}}\right)$. They are therefore just as or more important than the one-loop corrections to the matching coefficients $C_{i}(\mu)$ given in Eq. (33). Here we will apply the effective theory to the soft contributions and leave the hard spectator contributions for future investigation.

In Ref. [12], Charles et al. showed that in heavy to light decays, in which both the heavy and light quark interact solely via soft gluons, there are only three independent matrix elements. Charles et al. derived their result by combining HQET with LEET and using the fact that the HQET spinors describing heavy quarks, $h_{v}$ and the LEET spinors describing highly energetic quarks interacting with soft gluons, $\xi_{n}$, have only two independent components. Using the relations $\psi h_{v}=h_{v}$ and $\not \xi_{n}=0$ they showed that at leading order in $1 / E$ (where $E$ is the energy of the light meson) matrix elements of all hadronic currents in LEET are determined by only three independent functions. Unfortunately, LEET is not sufficient to describe heavy to light decays because it omits interactions with collinear gluons.

However, as pointed out in section II the spinors in the effective theory describing highly energetic quarks interacting with both soft and collinear gluons still have two components. In Eq. (27) we see that there are only four independent heavy to light currents in the collinear soft effective theory. For decays to pseudoscalar mesons like the $\pi$ and $K$ the matrix elements of these currents are

$$
\begin{aligned}
\left\langle P_{n}\left|\bar{\chi}_{n, P} h_{v}\right| H_{v}\right\rangle & =2 E \zeta(E), \\
\left\langle P_{n}\left|\bar{\chi}_{n, P} \gamma^{5} h_{v}\right| H_{v}\right\rangle & =0, \\
\left\langle P_{n}\left|\bar{\chi}_{n, P} \gamma_{\perp}^{\mu} h_{v}\right| H_{v}\right\rangle & =0,
\end{aligned}
$$

while for decays to vector mesons such as $\rho$ and $K^{*}$ they are

$$
\begin{aligned}
\left\langle V_{n}\left|\bar{\chi}_{n, P} h_{v}\right| H_{v}\right\rangle & =0, \\
\left\langle V_{n}\left|\bar{\chi}_{n, P} \gamma^{5} h_{v}\right| H_{v}\right\rangle & =-2 m_{V} \zeta_{\|}(E) v \cdot \epsilon^{*}, \\
\left\langle V_{n}\left|\bar{\chi}_{n, P} \gamma_{\perp}^{\mu} h_{v}\right| H_{v}\right\rangle & =2 E \zeta_{\perp}(E) i \epsilon_{\perp}^{\mu \nu} \epsilon_{\nu}^{*},
\end{aligned}
$$


where $\epsilon_{\perp}^{\mu \nu}=\epsilon^{\mu \nu \sigma \tau} v_{\sigma} n_{\tau}$ and we are using relativistic normalization for all effective theory states. Thus, there are still only three linearly independent soft form factors in the complete effective theory. Together with Eq. (27) these matrix elements determine that at tree level the heavy to light form factors are

$$
\begin{aligned}
& f_{+}\left(q^{2}\right)=\zeta(E), \quad f_{0}\left(q^{2}\right)=2 \hat{E} \zeta(E), \quad f_{T}\left(q^{2}\right)=\zeta(E), \\
& A_{1}\left(q^{2}\right)=2 \hat{E} \zeta_{\perp}(E), \quad A_{2}\left(q^{2}\right)=\zeta_{\perp}(E), \quad V\left(q^{2}\right)=\zeta_{\perp}(E), \\
& T_{1}\left(q^{2}\right)=\zeta_{\perp}(E), \quad T_{2}\left(q^{2}\right)=2 \hat{E} \zeta_{\perp}(E), \quad T_{3}\left(q^{2}\right)=\zeta_{\perp}(E), \\
& A_{0}\left(q^{2}\right)=\zeta_{\|}(E) \text {. }
\end{aligned}
$$

In deriving these relations we have dropped terms suppressed by $m_{P, V} / E$ since these corrections are expected to be just as large as $\lambda$ suppressed power corrections which are not included. Thus, $\zeta_{\|}(E)$ only appears in the purely longitudinal form factor $A_{0}\left(q^{2}\right)$. Taking this into account our results are in agreement with Ref. [12].

From the results in section $[1]$ we can obtain some more information on the heavy to light form factors. The results of Eqs. (27) and (33) determine the perturbative corrections to Eq. (59). Hard corrections do not break the symmetry relations between effective theory matrix elements, but do change the relation between form factors in the full and effective theory. We find

$$
\begin{aligned}
f_{+}\left(q^{2}\right) & =\zeta(E)\left[C_{4}+\hat{E} C_{5}\right], \\
f_{0}\left(q^{2}\right) & =\zeta(E) 2 \hat{E}\left[C_{4}+C_{5}(1-\hat{E})\right], \\
f_{T}\left(q^{2}\right) & =\zeta(E) C_{11}, \\
A_{1}\left(q^{2}\right) & =\zeta_{\perp}(E) 2 \hat{E} C_{3}, \\
A_{2}\left(q^{2}\right) & =\zeta_{\perp}(E) C_{3}, \\
V\left(q^{2}\right) & =\zeta_{\perp}(E) C_{3}, \\
T_{1}\left(q^{2}\right) & =\zeta_{\perp}(E) C_{9}, \\
T_{2}\left(q^{2}\right) & =\zeta_{\perp}(E) 2 \hat{E} C_{9}, \\
T_{3}\left(q^{2}\right) & =\zeta_{\perp}(E) C_{9}, \\
A_{0}\left(q^{2}\right) & =\zeta_{\|}(E)\left[C_{4}+C_{5}(1-\hat{E})\right] .
\end{aligned}
$$

where $C_{i}=C_{i}(2 \hat{E})$ and we have used the helicity relations given below Eq. (33). In Ref. 23] it was pointed out that the ratios, $V / A_{1}$ and $T_{1} / T_{2}$ do not receive perturbative corrections due to the fact that interactions which flip the helicity of the energetic quark are suppressed by $1 / E$. From Eq. (60) we see that in fact at leading order in $\lambda$ the soft contributions to the form factors $\left\{A_{1}, A_{2}, V\right\}$ and $\left\{T_{1}, T_{2}, T_{3}\right\}$ are related to all orders in $\alpha_{s}$. Furthermore, 
since the RGE's for all currents are identical any ratio of soft form factors are independent of Sudakov logarithms.

At one loop the hard corrections to ratios of the form factors in Eq. (55) were previously calculated in Ref. [13]. Since the authors used LEET as their effective theory their matching calculation was not infrared safe and the overall normalization of the low energy matrix elements was unknown. However, it was noted that this problem cancels out of ratios of form factors because the infrared divergences in the full theory are universal. Our results in Eq. (60) do not suffer from this problem because the collinear-soft effective theory has the same infrared divergences as QCD. Taking ratios of the form factors in Eq. (60), substituting the results in Eq. (33), and expanding in $\alpha_{s}\left(m_{b}\right)$, our results for the hard corrections to the soft form factors agree with those of Ref. [13].

As an application, consider the zero in the forward-backward asymmetry of the rare decay $B \rightarrow K^{*} \ell^{+} \ell^{-}$, which gives a relation between the Wilson coefficients $C_{9}^{\text {full }}$ and $C_{7}^{\text {full }}$ 24, 25]

$$
\operatorname{Re}\left[\frac{C_{9}^{\text {full }}\left(s_{0}\right)}{C_{7}^{\text {full }}}\right]=-\frac{m_{b}}{s_{0}}\left[\frac{T_{2}\left(s_{0}\right)}{A_{1}\left(s_{0}\right)}\left(m_{B}-m_{K^{*}}\right)+\frac{T_{1}\left(s_{0}\right)}{V\left(s_{0}\right)}\left(m_{B}+m_{K^{*}}\right)\right],
$$

where here $s_{0} \sim 3 \mathrm{GeV}$ is the value of $q^{2}$ where the asymmetry vanishes. It was noted in Ref. 25] that in the ratio of soft form factors the effective theory form factors cancel. Ignoring again the hard spectator contributions and the higher order effect of the mass of the $K^{*}$ we find

$$
\begin{aligned}
\operatorname{Re}\left[\frac{C_{9}^{\text {full }}\left(s_{0}\right)}{C_{7}^{\text {full }}}\right] & =-m_{B} \frac{m_{b}}{s_{0}}\left[\frac{2 C_{9}\left(m_{b}\right)}{C_{3}\left(m_{b}\right)}\right] \\
& =-2 m_{B} \frac{m_{b}}{s_{0}}\left(1+\frac{\alpha_{s}\left(m_{b}\right) C_{F}}{4 \pi} \ln (2 \hat{E}) \frac{2 \hat{E}}{1-2 \hat{E}}\right),
\end{aligned}
$$

where the perturbative correction from the soft form factor is in agreement with Ref. [13. There are additional order $\alpha_{s}$ corrections to Eq. (62) from collinear gluon exchange with the spectator in the B, which can be found in Ref. [13. Although Sudakov logarithms do not effect the ratio of purely soft form factors, they may suppress the soft contribution relative to that from collinear gluon exchange.

\section{CONCLUSIONS}

In this paper we investigated in detail the collinear-soft effective theory, which describes highly energetic particles with low invariant mass. The degrees of freedom in this theory consist of collinear quarks and gluons with momenta scaling as $k_{c}=Q\left(\lambda^{2}, 1, \lambda\right)$ and soft gluons with momenta scaling as $k_{s}=Q\left(\lambda^{2}, \lambda^{2}, \lambda^{2}\right)$. We gave a detailed derivation of the 
collinear-soft Lagrangian with the intent of making it straightforward to go to subleading orders in $\lambda$. In addition we derived the effective theory heavy-to-light current at order $\lambda^{0}$. For decays of heavy particles there are regions of phase space where this theory applies, namely when the hadronic decay products are light and are produced with large energy. The currents mediating these decays are given by four linearly independent operators in the effective theory. We performed the matching onto these operators at the one loop level and calculated their renormalization group evolution from the hard scale $Q \sim m_{b}$ to the intermediate scale $Q \lambda$.

We considered two applications of the collinear-soft theory: inclusive and exclusive decays. In the inclusive case we focused our attention on the radiative decay $B \rightarrow X_{s} \gamma$ and the semileptonic decay $B \rightarrow X_{u} \ell \bar{\nu}$ in the endpoint region of large photon energy and of low hadronic invariant mass, respectively. At leading order the OPE in the effective theory gives a bilocal operator whose matrix element is the universal nonperturbative light cone structure function of the $B$ meson. As is well known, in the ratio of large moments of these two decays this structure function cancels. As a consequence the Sudakov logarithms in this ratio are entirely determined by the running in the collinear-soft theory as discussed in Section $\nabla A$. Our result is in agreement with previous literature [9,10].

For exclusive decays we investigated the relationship amongst form factors in the large energy limit of QCD. In Ref. [12] it was shown using LEET that there are only three independent soft form factors at leading order in an expansion in inverse powers of the energy of the light quark. However, since LEET does not include collinear gluons it does not correctly reproduce the IR logarithms of QCD, and the relevance of this result is not immediately obvious. We showed that the presence of collinear gluons does not spoil the relations among the soft form factors, therefore establishing these results in the large energy limit of QCD. Finally we used the one loop matching of the currents in the effective theory to relate the full theory form factors to the three independent matrix elements in the effective theory. Our analysis confirms the corresponding results in Ref. [13], but with an infrared safe definition of the matching coefficients.

\section{ACKNOWLEDGMENTS}

We would like to thank Michael Luke for collaboration on early stages of this project and Ira Rothstein for numerous discussions. We would also like thank Mark Wise for discussion on the helicity invariance. C.B., S.F., and I.S. would like to thank the INT at the University of Washington for their hospitality at an early stage of this work. S.F. would like to thank the Caltech theory group for their hospitality while part of this work was completed. D.P. 
is grateful to the National Center of Theoretical Sciences, R.O.C. for hospitality during the final phase of this work. This work was supported in part by the Department of Energy under grants DOE-FG03-97ER40546 and DOE-ER-40682-143 and by NSERC of Canada.

\section{APPENDIX A: COLLINEAR GAUGE TRANSFORMATIONS}

In this appendix we discuss the collinear gauge invariance in the soft-collinear effective theory. For simplicity we will restrict ourselves to the abelian case. From the general set of gauge transformations $U(x)=e^{i \alpha(x)}$, where

$$
\psi(x) \rightarrow U(x) \psi(x), \quad A_{\mu}(x) \rightarrow A_{\mu}(x)-\frac{i}{g} U^{\dagger}(x) \partial_{\mu} U(x),
$$

the collinear transformations belong to a subset where $\partial_{\mu} \alpha(x)$ scales like a collinear momentum. To make this scaling explicit we decompose an arbitrary collinear gauge transformation as

$$
U(x) \equiv \int d^{4} Q e^{i Q \cdot x} \beta(Q)=\sum_{\tilde{Q}} e^{i \tilde{Q} \cdot x} \beta_{Q}\left(x^{-}\right)
$$

where $\left(\bar{n} \cdot Q, Q_{\perp}, n \cdot Q\right) \sim\left(\lambda^{0}, \lambda, \lambda^{2}\right)$ and the sum is over $\tilde{Q}=\left(\bar{n} \cdot Q, Q_{\perp}\right)$. For notational convenience we will suppress the dependence of $\beta_{Q}$ on $x^{-}$henceforth.

In Section II the full quark field was decomposed into components $\xi_{n, p}(x)$ which no longer depend on the large phases $e^{-i \tilde{p} \cdot x}$. Under the collinear gauge transformation in Eq. (A2) we have

$$
\sum_{\tilde{p}} e^{-i \tilde{p} \cdot x} \xi_{n, p}(x) \rightarrow \sum_{\tilde{p}} \sum_{\tilde{Q}} e^{-i(\tilde{p}-\tilde{Q}) \cdot x} \beta_{Q} \xi_{n, p}(x)=\sum_{\tilde{p}} e^{-i \tilde{p} \cdot x} \sum_{\tilde{Q}} \beta_{Q} \xi_{n, p+Q}(x) .
$$

Up to terms suppressed by powers of $\lambda$ the $x$ dependence of $\xi_{n, p}$ can be ignored and the Fourier components must agree, so

$$
\xi_{n, p}(x) \rightarrow \sum_{\tilde{Q}} \beta_{Q-p} \xi_{n, Q}(x) .
$$

Thus, the collinear gauge invariance simply corresponds to a "reparameterization" invariance of the theory under changes to the effective theory labels. Similarly, for the collinear gluon field with label $\tilde{q}$ we find

$$
\sum_{\tilde{q}} e^{-i \tilde{q} \cdot x} A_{n, q}^{\mu}(x) \rightarrow \sum_{\tilde{q}} e^{-i \tilde{q} \cdot x} A_{n, q}^{\mu}(x)+\frac{1}{g} \sum_{\tilde{R}} e^{-i \tilde{R} \cdot x} \sum_{\tilde{Q}} \beta_{R+Q}^{*}\left[\beta_{Q} Q^{\mu}-i \partial^{\mu} \beta_{Q}\right],
$$

so the components transform as 


$$
A_{q}^{\mu} \rightarrow A_{q}^{\mu}+\frac{1}{g} \sum_{\tilde{Q}} \beta_{Q+q}^{*}\left[Q^{\mu} \beta_{Q}-i \partial^{\mu} \beta_{Q}\right]
$$

Using the transformation properties ( $\overline{A 4}$ ) and ( $\overline{A 6}$ ) for the collinear quark and gluon field respectively, it is easy to see that the soft-collinear effective Lagrangian in Eq. (10) is gauge invariant. To see this, it is sufficient to note that the following combination of collinear fields transforms in the same manner as the collinear quark field in Eq. (A3),

$$
\sum_{\tilde{p}} e^{-i \tilde{p} \cdot x}\left(\tilde{p}^{\mu}+\frac{\bar{n}^{\mu}}{2} i n \cdot \partial+g \sum_{\tilde{q}} e^{-i \tilde{q} \cdot x} A_{n, q}^{\mu}\right) \xi_{n, p} .
$$

The derivation is somewhat tedious, so we will not display the details. However, we note that to derive this result it is necessary to make use of the unitarity of the gauge transformation, $U^{\dagger}(x) U(x)=1$ which implies

$$
\sum_{\tilde{P}, \tilde{Q}} \beta_{Q} \beta_{P}^{*} e^{i(Q-P) \cdot x}=1
$$

Finally, we show that the jet field $\chi_{n, P}$ from Section III,

$$
\chi_{n, P}=\sum_{\tilde{p}} e^{-i \tilde{p} \cdot x} \exp \left(\sum_{\tilde{q}} e^{-i \tilde{q} \cdot x} \frac{g \bar{n} \cdot A_{n, q}}{\bar{n} \cdot q}\right) \xi_{n, p},
$$

is invariant under the collinear gauge transformation in Eq. (A2). We have

$$
\chi_{n, P} \rightarrow \sum_{\tilde{p}} e^{-i p \cdot x} \exp \left[\sum_{\tilde{q}} e^{-i \tilde{q} \cdot x} \frac{g \bar{n} \cdot A_{n, q}}{\bar{n} \cdot q}+\sum_{\tilde{q}} \frac{e^{-i \tilde{q} \cdot x}}{\bar{n} \cdot q} \sum_{\tilde{Q}} \bar{n} \cdot Q \beta_{Q} \beta_{Q+q}^{*}\right] \sum_{R} \beta_{R-p} \xi_{n, R} .
$$

Comparing Eqs. (A1) and (A5) and using $\bar{n} \cdot \partial \beta_{Q}=0$ gives

$$
-i \bar{n} \cdot \partial \alpha(x)=\sum_{\tilde{R}, \tilde{Q}} e^{-i \tilde{R} \cdot x} \beta_{R+Q}^{*} \beta_{Q}(-i \bar{n} \cdot Q) .
$$

Integrating this result with respect to $n \cdot x / 2$ and taking the exponential gives the relation

$$
\exp \left[\sum_{\tilde{R}, \tilde{Q}} e^{-i \tilde{R} \cdot x} \beta_{R+Q}^{*} \beta_{Q} \frac{\bar{n} \cdot Q}{\bar{n} \cdot R}\right]=e^{-i \alpha(x)}=\sum_{\tilde{Q}} e^{-i \tilde{Q} \cdot x} \beta_{Q}^{*} .
$$

Substituting Eq. (A12) into Eq. (A10) and shifting $\tilde{p} \rightarrow \tilde{R}-\tilde{p}$ leaves

$$
\sum_{\tilde{R}} e^{-i \tilde{R} \cdot x} \exp \left[\sum_{\tilde{q}} e^{-i q \cdot x} \frac{g \bar{n} \cdot A_{n, q}}{\bar{n} \cdot q}\right] \sum_{\tilde{p}} e^{i \tilde{p} \cdot x} \beta_{p} \sum_{\tilde{Q}} e^{-i \tilde{Q} \cdot x} \beta_{Q}^{*} \xi_{n, R}=\chi_{n, P},
$$

where in the last step we have used the unitarity relation in Eq. (A8) and the definition in Eq. (A9). Thus, the jet field is invariant under a collinear gauge transformation as expected. 


\section{APPENDIX B: RENORMALIZATION OF THE CURRENT IN THE COLLINEAR-SOFT THEORY}

In Section III we quoted the renormalization constant of the current operator $\bar{\chi}_{n, P} \Gamma h_{v}$ in the collinear-soft theory

$$
Z=1+\frac{\alpha_{s} C_{F}}{4 \pi}\left(\frac{1}{\epsilon^{2}}+\frac{2}{\epsilon} \log \frac{\mu}{\bar{n} \cdot P}+\frac{5}{2 \epsilon}\right) .
$$

This was obtained by computing the renormalization of the term $\bar{\xi}_{n, p} \Gamma h_{v}$ which is the first term obtained using the expansion of $\bar{\chi}_{n, P}$ in Eq. (25). However, the renormalization constant (B1) depends only on the large component of the jet momentum $\bar{n} \cdot P$, which enters as a label on the jet field $\chi_{n, P}$. This is a nontrivial consequence of collinear gauge invariance and is essential for a consistent renormalization of the collinear-soft effective theory. For example, in the collinear diagram in Fig. 6]c the Wilson coefficient depends on only the sum of the collinear gluon and quark momentum in the loop. Thus, it depends only on $p$ and not on the loop momentum.

In this Appendix we illustrate this property of the current by explicit calculation of the corresponding renormalization of the one collinear gluon term in the expansion of $\bar{\chi}_{n, P} \Gamma h_{v}$. For simplicity we will work with an Abelian gauge theory (QED), for which this expansion has been given explicitly in Eq. (21) (with $P=p+\sum_{i} q_{i}$ for each term)

$$
\bar{\chi}_{n, P} \Gamma h_{v}=\bar{\xi}_{n, P} \Gamma h_{v}-\frac{g}{\bar{n} \cdot q} \bar{\xi}_{n, p} \bar{n} \cdot A_{n, q} \Gamma h_{v}+\frac{g^{2}}{\bar{n} \cdot q_{1} \bar{n} \cdot q_{2}} \bar{\xi}_{n, p} \bar{n} \cdot A_{n, q_{1}} \bar{n} \cdot A_{n, q_{2}} \Gamma h_{v}+\cdots
$$

The diagrams contributing to the renormalization of the second term in this expansion are shown in Figs. 7 and 8. We will work throughout in Feynman gauge. The diagrams in Fig. [7(a) and Fig. 8(a) have been computed already; the external gluon momentum $q$ does not enter the loop integral, so they can be simply extracted from the corresponding results for $\bar{\xi}_{n, P} \Gamma h_{v}$ (Eqs. (35), (36)):

$$
7(\mathrm{a})+8(\mathrm{a})=\left\langle-\frac{g}{\bar{n} \cdot q} \bar{\xi}_{n, p} \bar{n} \cdot A_{n, q} \Gamma h_{v}\right\rangle \cdot \frac{\alpha}{4 \pi}\left(\frac{1}{\epsilon^{2}}+\frac{2}{\epsilon}+\frac{2}{\epsilon} \log \frac{\mu}{\bar{n} \cdot p}+\text { const }\right) .
$$

Furthermore, upon examining the Feynman rule for the two collinear gluon coupling in Fig. 1, one can see that the graph in Fig. 8(c) vanishes in Feynman gauge. We will show in the following that the net effect of the two remaining graphs Fig. 7(b) and Fig. 8(b) is to change $\bar{n} \cdot p$ in the argument of the logarithm in (B3) to $\bar{n} \cdot(p+q)$, corresponding to the total momentum $P=p+q$ carried by the jet.

For simplicity we will take the external momenta $p, q$ to be off-shell and to have vanishing transverse components, $p=\left(p_{+}, p_{-}, 0_{\perp}\right)$ and $q=\left(q_{+}, q_{-}, 0_{\perp}\right)$. With this choice the soft diagram Fig. 7(b) reduces to one term, 
a)

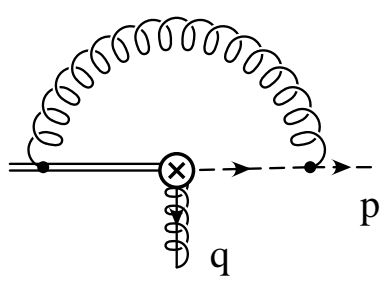

b)

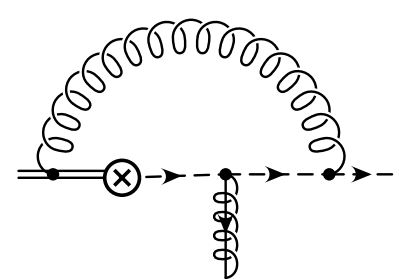

FIG. 7. One-gluon diagrams contributing to the soft gluon renormalization of the current operator $\bar{\chi}_{n} \Gamma h$ in an abelian gauge theory. The crossed dot denotes one insertion of the operator $\bar{\chi}_{n} \Gamma h_{v}$.

a)

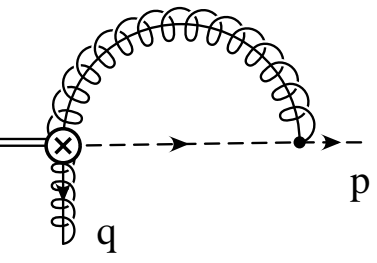

b)

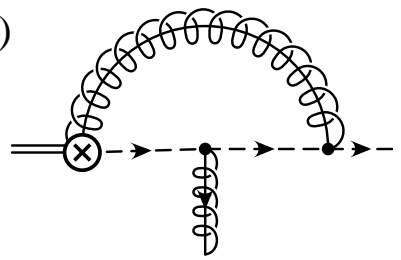

c)

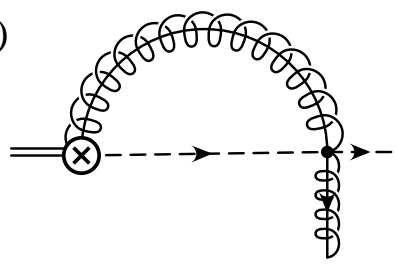

FIG. 8. Collinear gluon renormalization of the current operator $\bar{\chi}_{n} \Gamma h$ with one external collinear gluon. The crossed dot denotes one insertion of the operator $\bar{\chi}_{n} \Gamma h_{v}$.

$$
7(\mathrm{~b})=-i g^{3} \int \frac{\mathrm{d}^{d} l}{(2 \pi)^{d}} \frac{\left\langle\bar{\xi}_{n, p} n \cdot A_{n, q} \Gamma h_{v}\right\rangle \mu^{2 \epsilon}}{\left[n \cdot l-p^{2} / \bar{n} \cdot p\right]\left[n \cdot l-(p+q)^{2} / \bar{n} \cdot(p+q)\right][v \cdot l]\left[l^{2}\right]} .
$$

The integration is performed most easily in light-cone coordinates $l=\left(l^{+}, l^{-}, l_{\perp}\right)$, where the $l^{+}$integral can be done by the method of residues. We obtain

$$
7(\mathrm{~b})=\left\langle\bar{\xi}_{n, p} n \cdot A_{n, q} \Gamma h_{v}\right\rangle \frac{g^{3}}{8 \pi^{2}} \frac{1}{n \cdot q}\left\{\frac{1}{\epsilon} \ln \left[\frac{n \cdot(p+q)}{n \cdot p}\right]+\text { const }\right\} .
$$

Since this graph does not give a contribution to $\left\langle\bar{\xi}_{n, p} \bar{n} \cdot A_{n, q} \Gamma h_{v}\right\rangle$, it does not contribute to the renormalization of the current. However, the resulting divergence seems to require a new truly non-local operator. We will show that this contribution cancels in the sum of diagrams.

The collinear graph in Fig. 8(b) can be written as

$$
8(\mathrm{~b})=\left\langle\bar{\xi}_{n, p} n \cdot A_{n, q} \Gamma h_{v}\right\rangle I_{1}+\left\langle\bar{\xi}_{n, p} \bar{n} \cdot A_{n, q} \Gamma h_{v}\right\rangle I_{2}
$$

where

$$
\begin{aligned}
& I_{1}=2 i g^{3} \mu^{2 \epsilon} \int \frac{\mathrm{d}^{d} l}{(2 \pi)^{d}} \frac{\bar{n} \cdot(p+l) \bar{n} \cdot(p+q+l)}{\bar{n} \cdot l(l+p)^{2}(l+p+q)^{2} l^{2}}, \\
& I_{2}=-2 i g^{3} \mu^{2 \epsilon} \int \frac{\mathrm{d}^{d} l}{(2 \pi)^{d}} \frac{l_{\perp}^{2}}{\bar{n} \cdot l(l+p)^{2}(l+p+q)^{2} l^{2}} .
\end{aligned}
$$

Once again the integration is simplified by using the method of residues on the $l^{+}$integral. Explicitly, we find 


$$
\begin{aligned}
& I_{1}=-\frac{g^{3}}{8 \pi^{2}} \frac{1}{n \cdot q}\left\{\frac{1}{\epsilon} \ln \left[\frac{n \cdot(p+q)}{n \cdot p}\right]+\text { const }\right\}, \\
& I_{2}=-\frac{g}{\bar{n} \cdot q} \cdot \frac{\alpha}{4 \pi}\left\{\frac{2}{\epsilon} \ln \left[\frac{\bar{n} \cdot p}{\bar{n} \cdot(p+q)}\right]+\text { const }\right\} .
\end{aligned}
$$

Thus, the first term in $I_{1}$ cancels the UV divergence in Eq. (B5), as required. The divergent term in $I_{2}$ converts the label in Eq. (B3) from $\bar{n} \cdot p$ to $\bar{n} \cdot(p+q)$. As advertised, the remaining UV divergence depends only on the total jet momentum $P=p+q$ :

$$
7(a, b)+8(a, b, c)=\left\langle-\frac{g}{\bar{n} \cdot q} \bar{\xi}_{n, p} \bar{n} \cdot A_{n, q} \Gamma h_{v}\right\rangle \cdot \frac{\alpha}{4 \pi}\left(\frac{1}{\epsilon^{2}}+\frac{2}{\epsilon}+\frac{2}{\epsilon} \log \frac{\mu}{\bar{n} \cdot P}+\text { const }\right) .
$$

After adding the contributions from the heavy quark and collinear quark field wavefunction renormalization, we reproduce the renormalization constant $Z$ in Eq. (B1) (after taking the color factor $C_{F} \rightarrow 1$ ). With similar techniques we have also checked that this holds for the renormalization of the term in Eq. (B2) that contains two collinear gluon fields. As argued in section III, collinear gauge invariance forces all the terms in the sum in Eq. (B2) to be renormalized in the same way, with a Wilson coefficient which depends only on the total jet momentum. The explicit calculations in this Appendix agree with this result. 


\section{REFERENCES}

[1] N. Isgur and M.B. Wise, Phys. Lett. B232 (1989) 113; Phys. Lett. B237 (1990) 527.

[2] E. Eichten and B. Hill, Phys. Lett. B234, 511 (1990); H. Georgi, Phys. Lett. B240, 447 (1990).

[3] M. A. Shifman and M. B. Voloshin, Sov. J. Nucl. Phys. 41, 120 (1985); J. Chay, H. Georgi and B. Grinstein, Phys. Lett. B247, 399 (1990); I. I. Bigi, M. Shifman, N. G. Uraltsev and A. Vainshtein, Phys. Rev. Lett. 71, 496 (1993).

[4] M. J. Dugan and B. Grinstein, Phys. Lett. B255, 583 (1991).

[5] U. Aglietti, G. Corbo and L. Trentadue, Int. J. Mod. Phys. A14, 1769 (1999); C. Balzereit, T. Mannel and W. Kilian, Phys. Rev. D58, 114029 (1998).

[6] C. W. Bauer, S. Fleming and M. Luke, Phys. Rev. D63, 014006 (2001).

[7] M. E. Luke, A. V. Manohar and I. Z. Rothstein, Phys. Rev. D61, 074025 (2000).

[8] V. A. Smirnov, Phys. Lett. B404, 101 (1997); M. Beneke and V. A. Smirnov, Nucl. Phys. B522, 321 (1998); V. A. Smirnov and E. R. Rakhmetov, Theor. Math. Phys. 120, 870 (1999); V. A. Smirnov, Phys. Lett. B465, 226 (1999); For applications see: J. H. Kuhn, A. A. Penin and V. A. Smirnov, Eur. Phys. J. C17, 97 (2000); M. Beneke, G. Buchalla, M. Neubert and C. T. Sachrajda, Nucl. Phys. B591, 313 (2000).

[9] R. Akhoury and I. Z. Rothstein, Phys. Rev. D54, 2349 (1996); G. P. Korchemsky and G. Sterman, Phys. Lett. B340, 96 (1994).

[10] A. K. Leibovich, I. Low, and I. Z. Rothstein, Phys. Rev. D62, 014010 (2000).

[11] V. Chernyak and I. Zhitnitsky, Nucl. Phys. B345, 137 (1990).

[12] J. Charles, A. Le Yaouanc, L. Oliver, O. Pene and J. C. Raynal, Phys. Rev. D60, 014001 (1999).

[13] M. Beneke and T. Feldmann, Nucl. Phys. B592, 3 (2000).

[14] S. J. Brodsky, H. Pauli and S. S. Pinsky, Phys. Rept. 301, 299 (1998); R. Venugopalan, nucl-th/9808023. H. Leutwyler, Nucl. Phys. B76, 413 (1974).

[15] J. D. Bjorken, J. B. Kogut and D. E. Soper, Phys. Rev. D3, 1382 (1971); J. B. Kogut and D. E. Soper, Phys. Rev. D1, 2901 (1970).

[16] M. Luke and A. V. Manohar, Phys. Rev. D55, 4129 (1997); M. Luke and M. J. Savage, Phys. Rev. D57, 413 (1998).

[17] W. Zhang and A. Harindranath, Phys. Rev. D48, 4881 (1993).

[18] B. Grinstein, R. Springer and M. B. Wise, Phys. Lett. B202, 138 (1988); B. Grinstein, M. J. Savage and M. B. Wise, Nucl. Phys. B319, 271 (1989).

[19] J. C. Collins and D. E. Soper, Nucl. Phys. B193, 381 (1981); J. C. Collins, D. E. Soper and G. Sterman, Nucl. Phys. B261, 104 (1985); See J. C. Collins in Perturbative Quantum Chromodynamics, Ed. A. H. Mueller, 573 (1989) and references therein.

[20] I. I. Bigi, M. A. Shifman, N. G. Uraltsev and A. I. Vainshtein, Int. J. Mod. Phys. A9, 2467 (1994); M. Neubert, Phys. Rev. D49, 4623 (1994).

[21] A. K. Leibovich and I. Z. Rothstein, Phys. Rev. D61, 074006 (2000); A. K. Leibovich, I. Low and I. Z. Rothstein, Phys. Rev. D61, 053006 (2000).

[22] R. Akhoury, G. Sterman and Y. P. Yao, Phys. Rev. D50, 358 (1994).

[23] G. Burdman and G. Hiller, hep-ph/0011266.

[24] A. Ali, T. Mannel and T. Morozumi, Phys. Lett. B273, 505 (1991).

[25] G. Burdman, Phys. Rev. D57, 4254 (1998); A. Ali, P. Ball, L. T. Handoko and G. Hiller, Phys. Rev. D61, 074024 (2000). 